\title{
Membrane Remodeling and Stimulation of Aggregation Following $\alpha$-Synuclein Adsorption to Phosphotidylserine Vesicles
}

\author{
Supporting Information \\ Brandon M. Hoover ${ }^{*}$,Zhizhang Shen ${ }^{\dagger}$, Curran G. Gahan ${ }^{\dagger}$, David M. Lynn ${ }^{\dagger}$, Reid C. Van Lehn ${ }^{*}{ }^{\dagger}$, and \\ Regina M. Murphy ${ }^{*}, \dagger$ \\ *Biophysics Program, University of Wisconsin, Madison, WI 53706 \\ ${ }^{\dagger}$ Department of Chemical and Biological Engineering, University of Wisconsin, Madison, WI 53706
}

\section{SUPPLEMENTAL EXPERIMENTAL METHODS}

Carboxyfluorescein dye leakage. 5-6-carboxyfluorescein (CF) (100 mM) was used to measure liposome integrity as previously described ${ }^{1,2}$. Briefly, lipids were resuspended in quenched $\mathrm{CF}$ and samples were incubated at $40^{\circ} \mathrm{C}$ for 30 minutes. Each sample was sonicated for 30 minutes in an icewater bath using a probe-tip sonicator and centrifuged for 5 minutes at $10,000 \mathrm{x}$ g. Samples were frozen and thawed 5 times using an ethanol/dry ice bath and then extruded 21 times through a 100-nm polycarbonate membrane using an Avanti Mini-Extruder (Avanti Lipids) on a hot plate set to $40^{\circ} \mathrm{C}$. Vesicles were separated from free CF using Sephadex G-25 PD-10 columns (GE Healthcare). Dye leakage was monitored at an excitation of $470 \mathrm{~nm}$ and emission of $525 \mathrm{~nm}$ using a QuantaMaster spectrofluormeter (PTI, Birmingham, NJ). Vesicles $(100 \mu \mathrm{M})$ were mixed with aSyn in protein:lipid (P:L) molar ratios of 1:1, 1:10, and 1:100. Fluorescence measurements were recorded for 60 minutes. Triton X100 (Sigma) $(0.1 \% \mathrm{vol} / \mathrm{vol})$ was used to obtain the maximum fluorescence $\left(f_{\max }\right)$ at the end of data collection. The background fluorescence at time $0\left(f_{0}\right)$ and leakage in the absence of protein was subtracted from the raw data. Total percentage of dye leakage was calculated using the equation $f_{\text {normalized }}$ $=\left(f_{x}-f_{0}\right) /\left(f_{\max }-f_{0}\right) \times 100$.

Dynamic Light Scattering (DLS). aSyn $(150 \mu \mathrm{M})$ and $100 \mathrm{~nm}$ DLPS vesicles $(150 \mu \mathrm{M})$ in PBS were separately filtered through a $0.22-\mu \mathrm{m}$ syringe filter (Millipore) before mixing into a precleaned quartz cuvette. The cuvette was placed into a bath containing refractive index matching decahydronaphthalene (Sigma) and equilibrated to $23^{\circ} \mathrm{C}$. Measurements were taken at $90^{\circ}$ scattering angle using a Brookhaven BI-200SM and Innova 90C (Coherent, Inc.) $488 \mathrm{~nm}, 150 \mathrm{~mW}$ argon laser. Autocorrelation data were collected for 5 min following 24h of co-incubation. CONTIN fit to the autocorrelation function was used to determine a scattered intensity size distribution ${ }^{3}$.

Stewart Assay. Relative lipid concentrations in the supernatant and pellet were determined by the Stewart assay ${ }^{4}$. Briefly, $2 \mathrm{~mL}$ of ferrothiocyanate was mixed with $2 \mathrm{~mL}$ of chloroform. Samples were added and mixed vigorously and then centrifuged at $1000 \mathrm{rpm}$ for 5 minutes. To determine lipid concentrations a standard curve was generated from DOPS between $0.01 \mathrm{mg}$ and $0.1 \mathrm{mg}$ in duplicate, and the absorbance of samples was measured at $452 \mathrm{~nm}$ and compared. Supernatant and pellet fractions were measured at the same wavelength and the percentage of lipid in the supernatant and pellet was determined.

\section{SUPPLEMENTAL SIMULATION METHODS}

Coarse-Grained Molecular Dynamics Simulations. Coarse-grained molecular dynamics (MD) simulations were performed using the BMW MARTINI force field, which combines approximately four non-hydrogen atoms into single beads to model the interactions of protein, water, and lipids. Simulations were performed using GROMACS $2016^{5}$. Equations of motion were integrated using the leapfrog 
algorithm with a time step of 20 fs. All simulations used the force field cutoffs and parameters recommended by the BMW MARTINI developers.

Simulations were performed to model the adsorption of aSyn to DOPS and DLPS vesicles and planar bilayers. 2048 lipid molecules were used in all systems. The 140 amino acids of aSyn (PDB ID: 1XQ8) were represented by 272 coarse-grained beads. The secondary structure of aSyn was selected as a broken alpha helix, where the residues 3-37 and 45-92 were modeled as alpha helices and the other residues as random coils ${ }^{6}$. The secondary structure was constrained during the simulations because aSyn is known to maintain alpha-helical structures upon bilayer adsorption. The components of all systems are listed in Table S1.

We selected the BMW MARTINI force field because the original MARTINI force field lacks a dipole on its water molecules and assumes of a system-wide dielectric constant of 15, and thus is unreliable for highly charged systems like the anionic bilayers studied here ${ }^{7}$. Prior work has found that the polarizable water model used with MARTINI can also lead to spurious results for interfacial adsorption ${ }^{8,9}$. Consequently, we chose BMW MARTINI to more accurately model the electrostatic interactions between aSyn residues and charged lipids. Since the BMW MARTINI force field overestimates bilayer mechanical properties ${ }^{10}$, including the area compressibility modulus, bending rigidity, and line tension, we performed all simulations (unless otherwise noted) at an elevated temperature of $360 \mathrm{~K}$ to reduce the bending rigidity and enhance sampling. The bending rigidities of DOPS at $360 \mathrm{~K}$ and $300 \mathrm{~K}$ were calculated from simulations to be $8.4 \pm 1.1 \times 10^{-20} \mathrm{~J}$ and $9.4 \pm 1.7 \times 10^{-20} \mathrm{~J}$, respectively. The value at $360 \mathrm{~K}$ is similar to the experimental value of the bending rigidity of DOPC at $300 \mathrm{~K}$, which has been measured to be $8.22 \times 10^{-20} \mathrm{~J}$ and is estimated to be comparable to the bending rigidity of DOPS due to the similar tail groups $^{11}$. For DLPS, bending rigidities were calculated to be $7.6 \pm 0.6$ and $8.1 \pm 0.8 \times 10^{-20} \mathrm{~J}$ at $360 \mathrm{~K}$ and $300 \mathrm{~K}$, respectively. To our knowledge there are no experimental data for the bending rigidity of DLPS, although decreasing the tail length of lipids with PC head groups decreases experimentally determined bending rigidities in agreement with these calculations ${ }^{11}$.

To test the effect of temperature on vesicle elongation, we simulated the vesicle systems with 10 aSyn monomers adsorbed on the surface at temperatures ranging 300-360 K in $20 \mathrm{~K}$ intervals. For these simulations, we used the standard MARTINI force field with $10 \%$ of the water beads set as "antifreeze" beads to prevent spurious ion or water clustering. We quantify elongation based on the meridonal and equatorial eccentricities after $300 \mathrm{~ns}$ of simulation time, which we found to be sufficient for convergence at all temperatures. Table S2 shows both eccentricities as a function of temperature. The meridional eccentricities at $360 \mathrm{~K}$ are $0.76 \pm 0.02$ and $0.62 \pm 0.04$ for DLPS and DOPS, respectively, which are within error of the values obtained from simulations using BMW MARTINI $(0.78 \pm 0.01$ and $0.59 \pm 0.03$, respectively). These similar values obtained by the two force fields suggests that we can determine the effect of temperature by using the regular MARTINI force field, which is more computationally efficient than BMW MARTINI. The snapshots in Figure S11 further highlight that aSyn monomers accumulate at the poles of elongated DLPS vesicles but do not induce elongation or aggregate for DOPS vesicles in agreement with the BMW MARTINI simulations. The meridional eccentricities of DLPS are greater than those of DOPS for all temperatures (with no consistent differences in equatorial eccentricites). We also observe that the difference between the meridonal and equatorial eccentricities is consistently higher for DLPS than DOPS, although this difference is less pronounced at lower simulation temperatures. Together, these two observations indicate that the greater elongation of DLPS than DOPS vesicles is not dependent on the simulation temperature.

Planar bilayers were composed of 1024 DOPS or DLPS molecules in each leaflet and had initial dimensions of $25 \times 25 \times 25 \mathrm{~nm}^{3}$. A single aSyn monomer was initially placed $\sim 2 \mathrm{~nm}$ above the upper leaflet of the bilayer and associated counterions were added to solution. Each aSyn-bilayer system was equilibrated in the $N P T$ ensemble at 1 bar and $360 \mathrm{~K}$ using a Berendsen thermostat and a semi-isotropic Berendsen barostat ${ }^{12}$ for $200 \mathrm{~ns}$, followed by a production run of $700 \mathrm{~ns}$ using a Nosé-Hoover thermostat ${ }^{13,14}$ and a semi-isotropic Parrinello-Rahman barostat ${ }^{15,16}$ with time constants of 4 ps. The entire workflow was repeated twice for each bilayer composition, using different initial aSyn configurations. During these simulations, aSyn monomers partially inserted into the bilayer; insertion depths are listed in Table S3. To 
characterize key interactions that influence insertion, we generated maps (Figure S13) that count the timeaveraged number of contacts between aSyn residues and lipid head and tail groups. A contact was counted if a protein side chain bead was within $0.5 \mathrm{~nm}$ of either a lipid head group bead or lipid tail group bead. In both bilayers, the most probable interactions are between negatively charged lipid phosphate beads (PO4) and positively charged beads in the two helices of the protein (residue numbers 6, 10, 23, 43, $45,58,80,96$, and 97 which are all cationic lysine or arginine residues) as expected based on their opposing charges.

The initial configurations of the DOPS and DLPS vesicles were generated by inserting additional water molecules on the $\mathrm{x}$ - and $\mathrm{y}$ - boundaries of the equilibrated bilayers described above, resulting in $\mathrm{x}-\mathrm{y}$ dimensions of $35 \times 35 \mathrm{~nm}^{2}$. This procedure results in a free boundary between the bilayer and water. The aSyn monomer added for the aSyn-bilayer simulations was then removed and the bilayer was simulated for $150 \mathrm{~ns}$ in an anisotropic $N P T$ ensemble using a Berendsen thermostat and barostat at 1 bar and $300 \mathrm{~K}$, resulting in vesicle self-assembly. Self-assembly at a lower temperature ensures reasonable vesicle structural properties. The resulting DOPS and DLPS vesicle diameters were $\sim 9.6 \mathrm{~nm}$ and $\sim 8.9 \mathrm{~nm}$, respectively. 10 aSyn monomers were then evenly distributed on the outer layer of each vesicle. We also performed control simulations of vesicles in the absence of aSyn to identify effects associated with aSyn binding. The aSyn-vesicle systems were equilibrated in an anisotropic NPT ensemble at 1 bar and $300 \mathrm{~K}$ for $120 \mathrm{~ns}$ (Berendsen thermostat and barostat with time constants of $2 \mathrm{ps}$ ), followed by a production run in the NVT ensemble at $360 \mathrm{~K}$ for $300 \mathrm{~ns}$ using a Nosé-Hoover thermostat with a time constant of 4 ps. $300 \mathrm{~ns}$ was sufficient for vesicle structural parameters to plateau (Figure S12). All aSyn-vesicle systems were modeled twice, starting from different initial velocities and aSyn configurations.

Simulations were also performed to study the diffusion of individual aSyn monomers on the outer leaflet of an elongated DLPS vesicle. In these simulations, 9 of the 10 aSyn monomers (excluding one of the two highlighted monomers) and their associated counterions were removed from the final configuration of the aSyn-vesicle production trajectory, leaving a single bound aSyn monomer and elongated DLPS vesicle that was used as a new initial configuration (simulation components are listed as DLPS Vesicle 3 in Table S1). The two possible monomers which were retained in the simulations were selected based on their proximity to the center of the elongated vesicle. The two test monomers are drawn as pink and orange in Figure S15B. The elongated shape of the DLPS vesicle was maintained by restraining the phosphate beads at the two poles to their initial positions using a force constant of 1000 $\mathrm{kJ} /\left(\mathrm{mol} \mathrm{nm}^{2}\right)$ in each Cartesian direction. In the first replica of this protocol (using the pink monomer in Figure S15B), the first simulation was performed for $260 \mathrm{~ns}$ with 100 phosphate beads at each pole restrained (Figure S16A). After the aSyn monomer moved close to the restrained beads at one pole (Figure S16B), a second simulation was performed for 240 ns with 30 phosphate beads at each pole restrained (Figure S16C). Again, the aSyn monomer moved closer to the pole (Figure S16D). A third simulation was then conducted for $240 \mathrm{~ns}$ with 24 phosphate beads restrained (Figure S16E), and no further migration was observed. In the second replica of this protocol (using the orange monomer in Figure S15B), only one simulation was performed for 260 ns with 100 phosphate beads at each pole restrained (Figure S16G). This series of simulations indicates that aSyn monomers diffuse toward the higher curvature poles of the elongated vesicle independent of the number of restrained DLPS lipids. All diffusion simulations were performed in the $N V T$ ensemble at $360 \mathrm{~K}$ using a Nosé-Hoover thermostat with a time constant of $4 \mathrm{ps}$.

Simulation Analysis. Vesicle eccentricities: In order to calculate the eccentricities of vesicles, the lengths of the three principle axes of an ellipsoid were fitted using the coordinates of phosphate beads of the outer vesicle leaflet (Figure S12). The eccentricities from the last $150 \mathrm{~ns}$ of the aSyn-vesicle production trajectories were averaged to obtain the values reported in the main text and Figure S12.

aSyn insertion depth: The insertion depth of an aSyn monomer on a planar DLPS or DOPS bilayer (Table S3) was defined as the z-component of the distance between the center of mass (COM) of the first 99 residues of aSyn and the COM of the phosphate beads of the upper leaflet. 
Bilayer height: The midplane height of a planar bilayer, $h(x, y)$, was defined based on the $z$ positions of the phosphate beads. For each simulation configuration, the $z$-positions of lipids in the upper and lower leaflets were interpolated using cubic splines onto a square grid (each point separated by 0.2 $\mathrm{nm}$ ) that spanned the $x-y$ plane. The midplane height was then defined at each grid point as the average of the upper and lower leaflet $z$-positions. To maintain a consistent coordinate system relative to a bound aSyn monomer, the COM of the first 99 residues of aSyn were defined as the origin of the coordinate system and the coordinates of all beads were rotated for each simulation configuration to align the long axis of the monomer with the $x$-axis. The midplane height was then time-averaged using the last $200 \mathrm{~ns}$ of the aSyn-bilayer production trajectories and used to generate the height contour maps in Figure S14, as well as the insertion depth and area per lipid in Table S3.

Bending rigidity: The bending rigidities of the DLPS and DOPS bilayers were calculated in the absence of aSyn from the power spectrum of the height fluctuations $\left\langle|h(\boldsymbol{q})|^{2}\right\rangle$, which is given by $\left\langle|h(\boldsymbol{q})|^{2}\right\rangle=\frac{k_{B} T}{K_{c} q^{4}}$, where $h$ is the bilayer surface height, $\boldsymbol{q}$ is the wavenumber in $\mathrm{nm}^{-1}, K_{c}$ is the bending rigidity, $k_{B}$ is the Boltzmann's constant, and $T$ is the temperature ${ }^{17}$. The values of $q^{4}\left\langle|h(\boldsymbol{q})|^{2}\right\rangle$ with wavelength greater than twice the bilayer thickness $(>6.8 \mathrm{~nm}$ and $>9.8 \mathrm{~nm}$ for DLPS and DOPS, respectively) were averaged to determine the $K_{c}$.

\section{Supplemental Analysis of Particle Shapes}

Spheres. Scattering from liposomes is well described by $P(q)_{\text {sphere }}$ of a solid sphere with radius $R$ $<100 \mathrm{~nm}^{18}$, given by the expression:

$$
P(q)_{\text {sphere }}=9\left[\frac{\sin x-x \cos x}{x^{3}}\right]^{2}
$$

where $x=q R, q=(4 \pi n / \lambda) \sin (\theta / 2), n=$ solvent refractive index, $\lambda=$ wavelength of incident beam and $\theta=$ scattering angle. We used $\theta=90^{\circ}$ for all calculations; for NTA the scattering angle is between 70 and $100^{\circ}{ }^{19}$.

Prolate ellipsoids. aSyn:DOPS complexes were modeled as prolate ellipsoids. The equivalent hydrodynamic diameter $D_{\text {sphere }}$ for prolate ellipsoids was calculated from ${ }^{20}$ :

$$
D_{\text {sphere }}=\frac{l \sqrt{r^{2}-1}}{r} \frac{1}{\ln \left[r+\sqrt{r^{2}-1}\right]} \text { for }(\mathrm{r}>1)
$$

where $r$ and $l$ represent the axial ratio (major axis to minor axis) and major axis of the ellipsoid, respectively. $P(q)$ for ellipsoids is given by ${ }^{21}$ :

$$
P(q)_{\text {ellipsoid }}=\int_{0}^{\pi / 2}\left[\Phi\left(q R^{\prime}\right)\right]^{2} \sin (\alpha) d \alpha
$$

where

$$
R^{\prime}=R\left(\sin ^{2} \alpha+l^{2} \cos ^{2} \alpha\right)^{1 / 2}
$$

and

$$
\Phi\left(q R^{\prime}\right)=\frac{3\left[\sin q R^{\prime}-q R^{\prime} \cos q R^{\prime}\right]}{q R^{\prime 3}}
$$


where $R$ is the equatorial radius, $l$ is the axial ratio, and $\alpha$ is the angle of integration.

Discoids. Discoid particles were observed in TEM images of mixtures of aSyn with DLPS. The diameter $d_{d i s k}$ and thickness $t$ of 47 particles were measured, yielding a distribution centered around $d_{d i s k}=$ $33 \pm 8$ and $t=6 \pm 3 \mathrm{~nm}$. The equivalent hydrodynamic diameter of discoids was calculated from ${ }^{22,23}$ :

$$
D_{\text {sphere }}=\frac{3 d_{\text {disk }}}{4}\left\{\left[1+\alpha^{2}\right]^{1 / 2}+\frac{1}{\alpha} \ln \left[\alpha+\left[1+\alpha^{2}\right]^{1 / 2}\right]-\alpha\right\}^{-1}
$$

where $\alpha=t / d_{\text {disk }}$, yielding a distribution with a mean value of $27 \pm 7 \mathrm{~nm}$ (Figure S10B).

Tubules. Tubules, observed in TEM images of mixtures of aSyn with DLPS, were typically 10-20 $\mathrm{nm}$ in diameter with lengths ranging between 300-700 $\mathrm{nm}$ (Figure 5D). We checked whether DLPS tubules could have been produced by remodeling of a single $100 \mathrm{~nm}$ LUV. The number of lipid molecules in a cylindrical body capped by two hemispheres is:

$$
N_{\text {lipid }}=\frac{2 \pi r L+2 \pi(r-h) L+4 \pi r^{2}+4 \pi(r-h)^{2}}{a}
$$

where $r$ is the radius, $L$ is the length of the cylindrical body, $h$ is the bilayer thickness, and $a$ is the area per lipid molecule, respectively. For a DLPS tubule of $L=700 \mathrm{~nm}$ and $r \sim 5 \mathrm{~nm}$ (Figure 5D), $N_{\text {lipid }}=5 \mathrm{x}$ $10^{4}$, equivalent to a spherical liposome of $72 \mathrm{~nm}$ in diameter (Eq. S1).

For estimating hydrodynamic properties, tubules were modeled as semiflexible (wormlike) chains. We assumed a chain diameter of $d_{c}=10 \mathrm{~nm}$ and estimated the persistence length by TEM to be $\sim 35 \mathrm{~nm}$. For wormlike chains, the Kuhn statistical length $l_{k}$ is double the persistence length, or $l_{k}=70$ $\mathrm{nm}$. Contour lengths, $L_{c}$, were measured for 13 particles and ranged from 300-700 nm. $D_{\text {sphere }}$ for semiflexible chains was calculated as described ${ }^{24,25}$ :

$$
\frac{L_{C}}{D_{\text {sphere }}}=A_{1}\left(\frac{L_{C}}{l_{k}}\right)^{0.5}+A_{2}+A_{3}\left(\frac{L_{C}}{l_{k}}\right)^{-0.5}+A_{4}\left(\frac{L_{C}}{l_{k}}\right)^{-1}+A_{5}\left(\frac{L_{C}}{l_{k}}\right)^{-1.5}
$$

for $L_{C} / l_{k}>2.278$. The coefficients $A_{i}$ are given by Yamakawa and Fuiji ${ }^{25}$.

The calculated value for $D_{\text {sphere }}$ ranged from $60-100 \mathrm{~nm}$, in good agreement with the size distribution measured by NTA. $P(q)$ for a semiflexible chain is given as ${ }^{26,27}$ :

$$
P(q)_{\text {tubule }}=\frac{2}{L_{c}} \int_{0}^{L_{c}}\left(L_{c}-t\right) \times \exp \left(-\frac{1}{6} q^{2} l_{k} \times t f(t)\right) \times \frac{\sin (q \times \operatorname{tg}(t))}{q \times \operatorname{tg}(t)} d t
$$

where the functions $f(t)$ and $g(t)$ are defined elsewhere ${ }^{27}$. We calculated a $P(q)_{\text {tubule }}$ between 0.36 and 0.64 for this length range between contour lengths of 700-300 nm.

Cylindrical particles. Some large complex structures including helical and twisted ribbons were observed in aSyn-DLPS mixtures. We modeled them simply as long cylindrical particles, which are well described by Broersma's relations ${ }^{28}$.

$$
D_{T}=\frac{k_{B} T}{3 \pi \eta L}\left[\delta-(1 / 2)\left(\gamma_{1}+\gamma_{2}\right)\right]
$$




$$
\begin{aligned}
& \gamma_{1}=0.807+\frac{0.15}{\delta}+\frac{13.5}{\delta^{2}}-\frac{37}{\delta^{3}}+\frac{22}{\delta^{4}} \\
& \gamma_{2}=-0.194+\frac{0.15}{\delta}+\frac{8.1}{\delta^{2}}-\frac{18}{\delta^{3}}+\frac{9}{\delta^{4}}
\end{aligned}
$$

$$
\text { with } \delta=\ln (2 L / d)
$$

and the Stokes-Einstein relationship:

$$
D_{\text {sphere }}=\frac{k_{B} T}{3 \pi \eta D_{t}}
$$

where $L$ is the cylinder length, $d$ is the diameter, $k_{B}$ is the Boltzmann constant, $T$ is the temperature and $\eta$ is the solvent viscosity. For a representative structure shown in Figure 5A, we measured $L \sim 1500 \mathrm{~nm}$ and $d \sim 70 \mathrm{~nm}$, corresponding to $D_{\text {sphere }}=374 \mathrm{~nm}$. To determine the shape factor, we modeled cylindrical particles using an expression for hollow cylinders ${ }^{29}$ :

$$
\begin{aligned}
P(q)_{\text {ribbbon }}= & \int_{0}^{1}\left(\frac{1}{1-\left(R_{2} / R_{1}\right)}\right)^{2}\left[\frac{2 J_{1}\left(q R_{1}\left(1-t^{2}\right)^{1 / 2}\right)}{q R_{1}\left(1-t^{2}\right)^{1 / 2}}\right. \\
& \left.-\frac{\left(R_{2} / R_{1}\right)^{2} 2 J_{1}\left(q R_{1}\left(1-t^{2}\right)^{1 / 2}\right)}{q R_{2}\left(1-t^{2}\right)^{1 / 2}}\right]^{2}\left(\frac{\sin (q L t / 2)}{q L t / 2}\right)^{2} d t
\end{aligned}
$$

Where $R_{1}, R_{2}$, and $L$ are the inner, outer radius and length of the cylinder, respectively, and $J_{l}(t)$ is the Bessel function of the first order. While it is difficult to estimate the thickness of the ribbons, by TEM they are thin $(\sim 5 \mathrm{~nm})$ by measuring the edge of the tip of the helical ribbon shown in Figure 5A. Thus, we estimated that $R_{1}$ and $R_{2}$ were approximately 30 and $35 \mathrm{~nm}$, respectively. Using a length of $\sim 1500 \mathrm{~nm}$, we calculated a $P(q)_{\text {ribbon }}$ of 0.01 . Hollow cylinders scatter weakly for their size given their extended shape and water filled interior. 


\section{Supporting Tables and Figures}

Table S1: Number of molecules of lipid, aSyn, water, $\mathrm{Na}+$, and $\mathrm{Cl}-$ in each bilayer and vesicle system, as well as simulation run times.

\begin{tabular}{|c|c|c|c|c|c|c|c|c|c|}
\hline & \multirow[b]{2}{*}{$\begin{array}{c}\text { DOPS } \\
\text { Bilayer } \\
1\end{array}$} & \multirow[b]{2}{*}{$\begin{array}{c}\text { DOPS } \\
\text { Bilayer } \\
2\end{array}$} & \multicolumn{4}{|c|}{ System } & \multirow[b]{2}{*}{$\begin{array}{c}\text { DOPS } \\
\text { Vesicle } \\
\text { (no } \\
\text { aSyn) } \\
\end{array}$} & \multirow[b]{2}{*}{$\begin{array}{c}\text { DOPS } \\
\text { Vesicle } \\
1 / 2\end{array}$} & \multirow[b]{2}{*}{$\begin{array}{c}\text { DLPS } \\
\text { Vesicle } \\
3\end{array}$} \\
\hline & & & $\begin{array}{c}\text { DLPS } \\
\text { Bilayer } \\
1\end{array}$ & $\begin{array}{c}\text { DLPS } \\
\text { Bilayer } \\
2\end{array}$ & $\begin{array}{c}\text { DLPS } \\
\text { Vesicle } \\
\text { (no } \\
\text { aSyn) }\end{array}$ & $\begin{array}{c}\text { DLPS } \\
\text { Vesicle } \\
1 / 2\end{array}$ & & & \\
\hline DOPS & 2048 & 2048 & 2048 & 2048 & 2048 & 2048 & 2048 & 2048 & 2048 \\
\hline$\alpha-S y n$ & 1 & 1 & 1 & 1 & 0 & 10 & 0 & 10 & 1 \\
\hline Water & 98662 & 98674 & 105560 & 105557 & 220259 & 219532 & 202368 & 201243 & 219532 \\
\hline $\mathrm{Na}+$ & 2485 & 2485 & 2587 & 2587 & 2578 & 2668 & 2476 & 2566 & 2587 \\
\hline $\mathrm{Cl}-$ & 428 & 428 & 530 & 530 & 530 & 530 & 428 & 428 & 530 \\
\hline $\begin{array}{l}\text { Equilibration } \\
\text { time (ns) }\end{array}$ & 200 & 200 & 200 & 200 & 120 & 120 & 120 & 120 & N/A \\
\hline $\begin{array}{l}\text { Production } \\
\text { Time (ns) }\end{array}$ & 700 & 700 & 700 & 700 & 300 & 300 & 300 & 300 & $260-740$ \\
\hline
\end{tabular}

Table S2: Effect of temperature on eccentricity.

\begin{tabular}{|c|c|c|c|}
\hline Lipid Type & $\begin{array}{c}\text { Temperature } \\
(\mathbf{K})\end{array}$ & Meridional & Equatorial \\
\hline DLPS & 300 & $0.51 \pm 0.06$ & $0.38 \pm 0.09$ \\
\hline DLPS & 320 & $0.45 \pm 0.06$ & $0.30 \pm 0.08$ \\
\hline DLPS & 340 & $0.64 \pm 0.03$ & $0.51 \pm 0.09$ \\
\hline DLPS & 360 & $0.76 \pm 0.02$ & $0.37 \pm 0.09$ \\
\hline DOPS & 300 & $0.45 \pm 0.07$ & $0.33 \pm 0.09$ \\
\hline DOPS & 320 & $0.38 \pm 0.06$ & $0.27 \pm 0.08$ \\
\hline DOPS & 340 & $0.51 \pm 0.05$ & $0.36 \pm 0.09$ \\
\hline DOPS & 360 & $0.62 \pm 0.04$ & $0.38 \pm 0.09$ \\
\hline
\end{tabular}


Table S3: aSyn insertion depth, area per lipid, and bending rigidity of each lipid bilayer systems.

\begin{tabular}{|c|c|c|c|c|c|}
\hline Lipid Type & System & $\begin{array}{c}\text { Temperature } \\
\text { (K) }\end{array}$ & $\begin{array}{c}\text { Insertion } \\
\text { Depth (nm) }\end{array}$ & $\begin{array}{c}\text { Area per } \\
\text { Lipid }\left(\mathrm{nm}^{2}\right)\end{array}$ & $\begin{array}{c}\text { Bending } \\
\text { Rigidity }\left(10^{-20} \mathrm{~J}\right)\end{array}$ \\
\hline \multirow{2}{*}{ DLPS } & Replica 1 & \multirow{4}{*}{360} & $-0.22 \pm 0.34$ & $0.66 \pm 0.002$ & $7.95 \pm 0.83$ \\
\hline & Replica 2 & & $0.27 \pm 0.28$ & $0.66 \pm 0.002$ & $7.26 \pm 0.29$ \\
\hline \multirow{2}{*}{ DOPS } & Replica 1 & & $0.26 \pm 0.27$ & $0.70 \pm 0.00$ & $8.42 \pm 1.08$ \\
\hline & Replica 2 & & $0.48 \pm 0.27$ & $0.70 \pm 0.00$ & $8.35 \pm 1.20$ \\
\hline \multirow{2}{*}{ DLPS } & Replica 1 & \multirow{4}{*}{300} & $0.08 \pm 0.21$ & $0.60 \pm 0.002$ & $8.69 \pm 0.98$ \\
\hline & Replica 2 & & $0.02 \pm 0.22$ & $0.60 \pm 0.002$ & $7.48 \pm 0.54$ \\
\hline \multirow{2}{*}{ DOPS } & Replica 1 & & $-0.01 \pm 0.23$ & $0.64 \pm 0.002$ & $11.25 \pm 2.01$ \\
\hline & Replica 2 & & $0.21 \pm 0.16$ & $0.64 \pm 0.002$ & $7.57 \pm 1.47$ \\
\hline
\end{tabular}

Table S4: Distribution of lipid and protein between supernatant and pellet following ultracentrifugation. Samples were prepared in PB at 1:10 P:L molar ratio and incubated for $48 \mathrm{~h}$ at $30^{\circ} \mathrm{C}$ before separation by ultracentrifugation.

\begin{tabular}{ccccc}
\hline & \multicolumn{2}{c}{ Lipid } & \multicolumn{2}{c}{ Protein } \\
\cline { 2 - 5 } Lipid & Supernatant & Pellet & Supernatant & Pellet \\
\hline \multirow{2}{*}{ DOPS } & $26 \%$ & $74 \%$ & $50 \%$ & $50 \%$ \\
& & & & \\
DMPS & undetectable & $100 \%$ & $29 \%$ & $71 \%$ \\
& & & & \\
DLPS & $91 \%$ & $9 \%$ & $87 \%$ & $13 \%$ \\
\hline
\end{tabular}




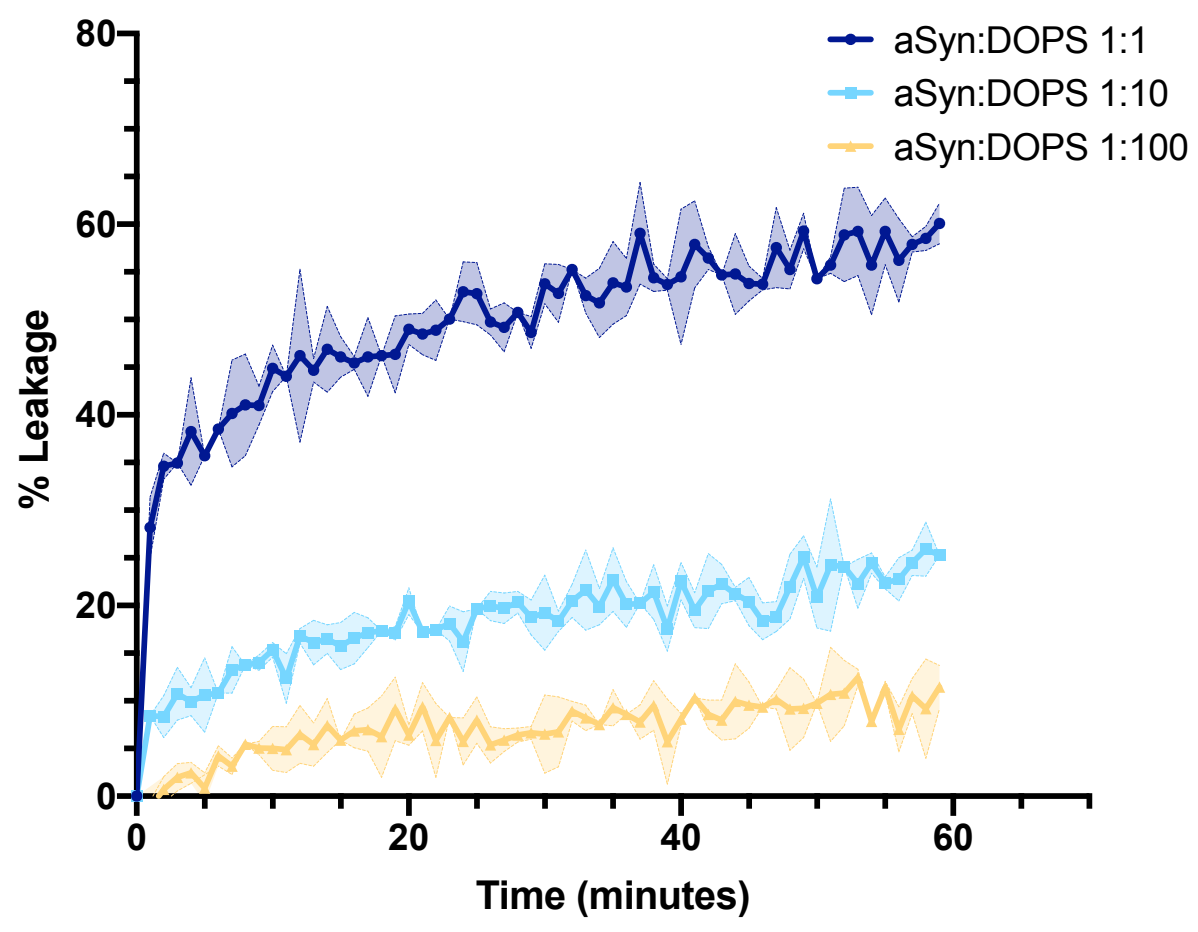

Figure S1: Carboxyfluorescein leakage from DOPS LUVs by aSyn. Samples were prepared at 1:100 (yellow), 1:10 (light blue) or 1:1 (blue) P:L molar ratio in PBS. Dye leakage was monitored by fluorescence. Baseline leakage from DOPS vesicles in the absence of protein was subtracted and the data were normalized to maximum fluorescence released with Triton X-100. Data points indicate the average of three replicates and shading indicates the standard deviation. 

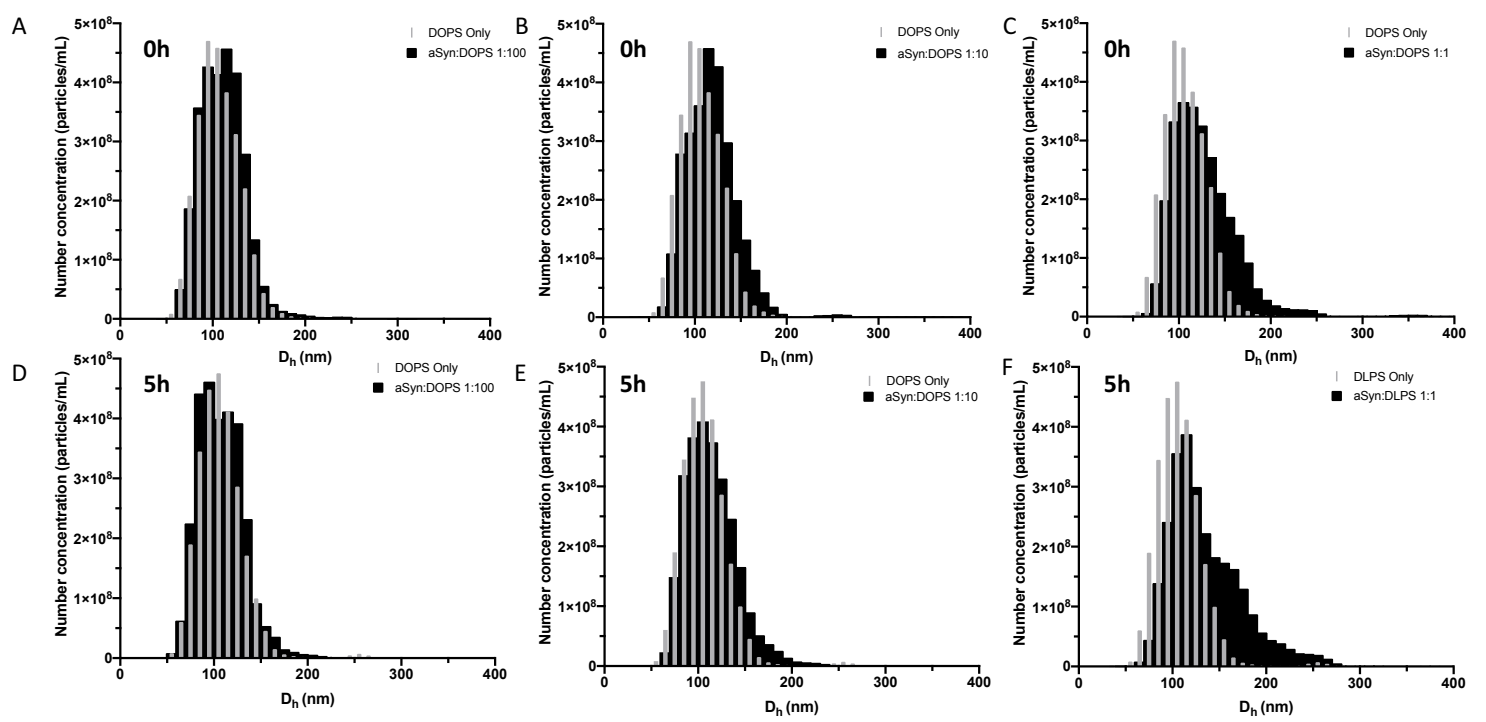

Figure S2: Size distributions for DOPS LUVs mixed with aSyn. Samples were prepared with aSyn and $100 \mathrm{~nm}$ DOPS LUVs at 1:100, 1:10 or 1:1 P:L molar ratios in PBS. Particle-by-particle size distributions were collected by NTA immediately after preparation and after $5 \mathrm{~h}$ incubation at room temperature. Mixtures of aSyn and DOPS (black) are compared to DOPS liposomes only (grey). The size distribution shifted towards greater hydrodynamic diameter with increasing protein and increasing time. Total number concentration remained essentially constant over $5 \mathrm{~h}$ (Figure 3B). All plots shown are representative distributions of a single replicate from three independent replicates.


Figure S3: NTA of $50 \mathrm{~nm}$ (nominal) DOPS LUVs with aSyn in PBS. (A) Mean hydrodynamic diameter of $50 \mathrm{~nm}$ DOPS vesicles mixed with aSyn at 1:100, 1:10 or 1:1 P:L molar ratio. Data are normalized to vesicles alone at $t=0$. The mean diameter of vesicles of three independent replicates was $65 \pm 5 \mathrm{~nm}$. $D_{\text {sphere }}$ increased by $2 \pm 1 \mathrm{~nm}, 5 \pm 3 \mathrm{~nm}$, and $9 \pm 1 \mathrm{~nm}$ for P:L of $1: 100,1: 10$, and $1: 1$, respectively. These increases were smaller for $50 \mathrm{~nm}$ liposomes than for $100 \mathrm{~nm}$ liposomes. If a monolayer of adsorbed aSyn were responsible for the increase in $D_{\text {sphere }}$, we would have expected the same size increase independent of the LUV diameter. (B) Particle number concentration of $50 \mathrm{~nm}$ DOPS vesicles mixed with aSyn at 1:100, 1:10 or 1:1 P:L molar ratio. Data are normalized to vesicles alone at $t=0$. Data points are the average of 3 independent replicates and shading indicates standard deviation. As with $100 \mathrm{~nm}$ LUVs, there was no significant change in liposome number. 

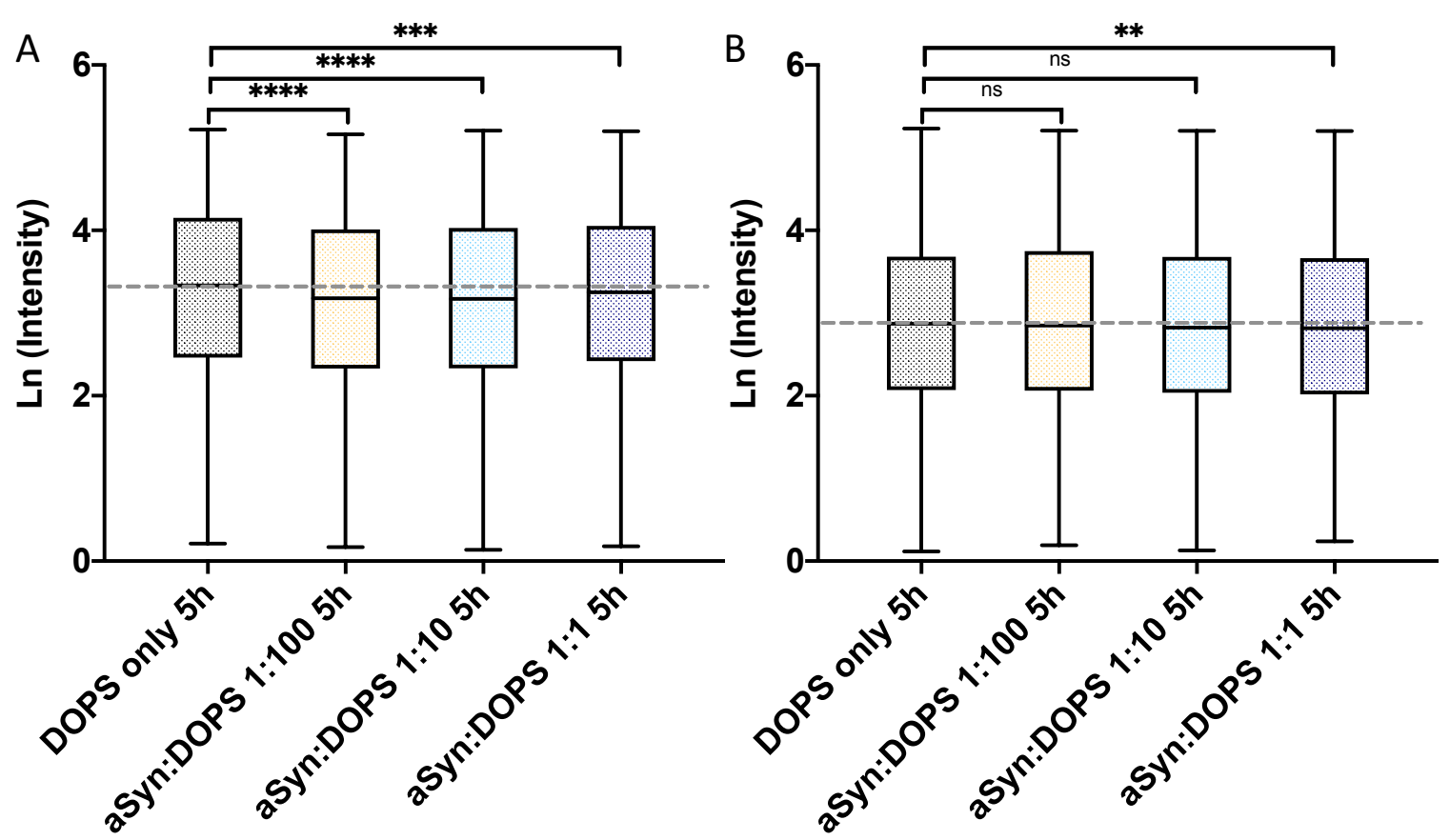

Figure S4: Box and whiskers plot of the scattered intensity distribution for size range between $75-125 \mathrm{~nm}$ following $5 \mathrm{~h}$ of co-incubation with DOPS vesicles for two additional independent replicates. (A) Approximately 7,200 DOPS vesicles in the absence of lipid were tracked and compared with 7,400 (P:L 1:100), 7,300 (P:L 1:10), and 5,600 (P:L 1:1) particles. (B) About 7,000 DOPS vesicles in the absence of lipid were tracked and compared with 7,800 (P:L 1:100), 7,700 (P:L 1:10), and 7,800 (P:L 1:1) particles. $n s$ not significant, ${ }^{* *} p<0.01,{ }^{* * *} p<0.001$, and $* * * * p<0.0001$, unpaired two-tailed t test. 


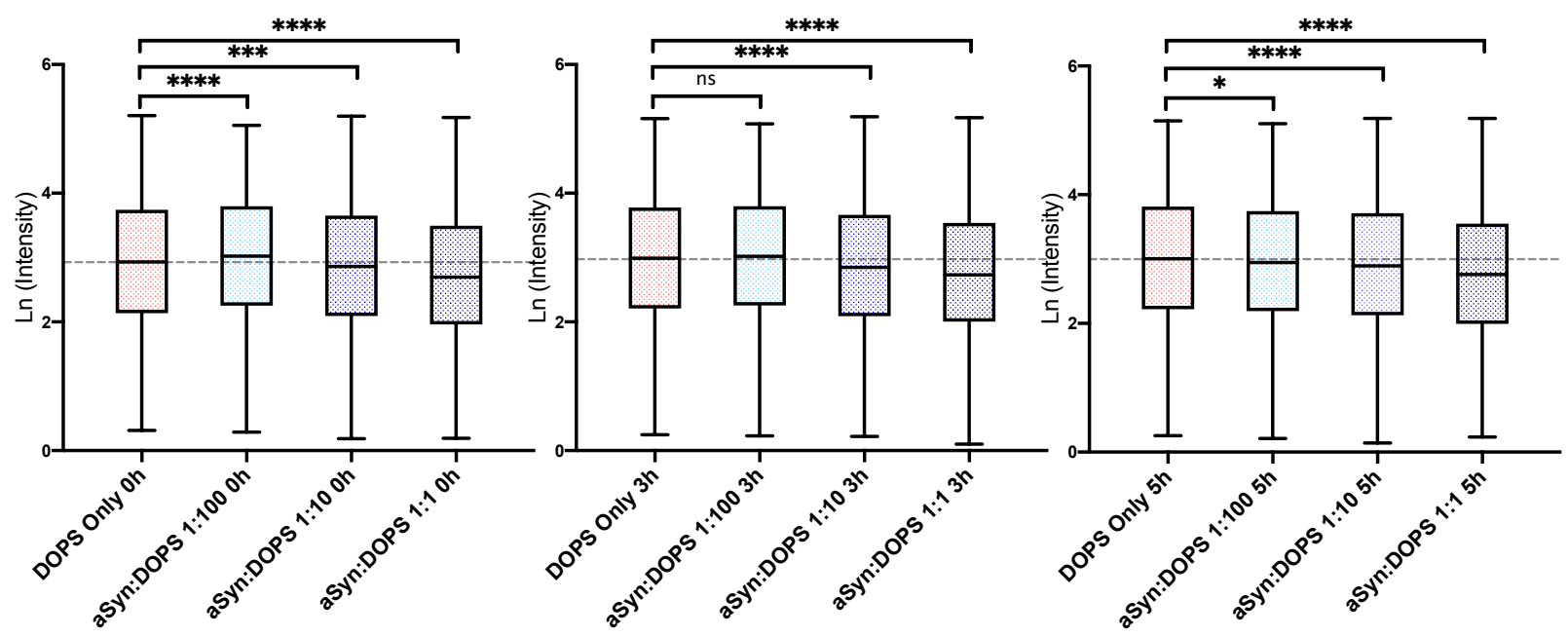

Figure S5: Box and whiskers plots showing scattered intensity distribution per particle for DOPS alone compared with aSyn added at P:L molar ratios of $1: 100,1: 10$, and $1: 1$ at $0 \mathrm{~h}, 3 \mathrm{~h}$ and $5 \mathrm{~h} . n s$, not significant, $* p<0.05, * * * p<0.001$, and $* * * * p<0.0001$ (unpaired two-tailed t test). Dotted line shows the mean scattered intensity per particle for DOPS vesicles in the absence of added protein. All plots shown are representative distributions of a single replicate from three independent replicates. 

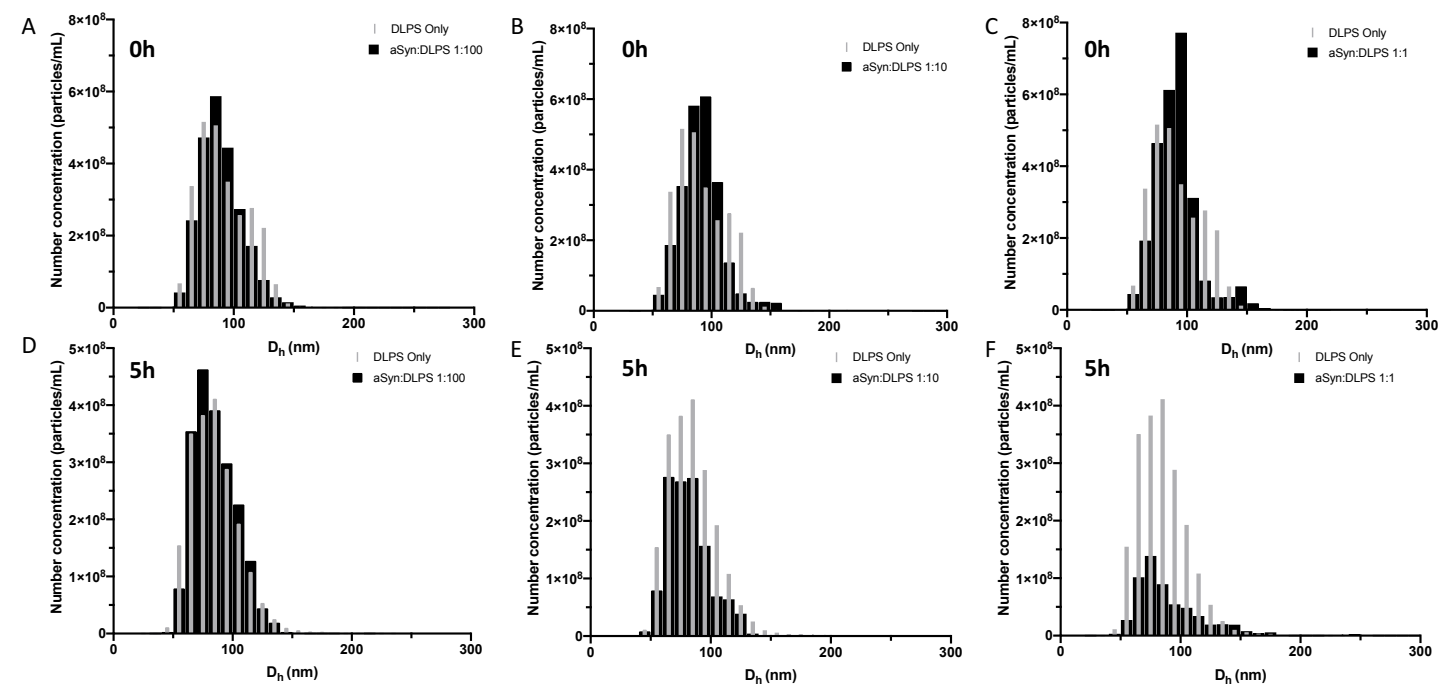

Figure S6: Size distributions for DLPS LUVs mixed with aSyn. Samples were prepared with aSyn and $100 \mathrm{~nm}$ DLPS LUVs at 1:100, 1:10 or 1:1 P:L molar ratios in PBS. Particle-by-particle size distributions were collected by NTA immediately after preparation and after $5 \mathrm{~h}$ incubation at room temperature. Mixtures of aSyn with DLPS (black) are compared to DLPS liposomes only (gray). The total number concentration of DLPS LUVs decreased over time following protein addition. The mean hydrodynamic diameter was approximately invariant with protein concentration and time, although at the highest P:L ratio the distribution became somewhat broader with the appearance of a few very large particles. All plots shown are representative distributions of a single replicate from three independent replicates. 

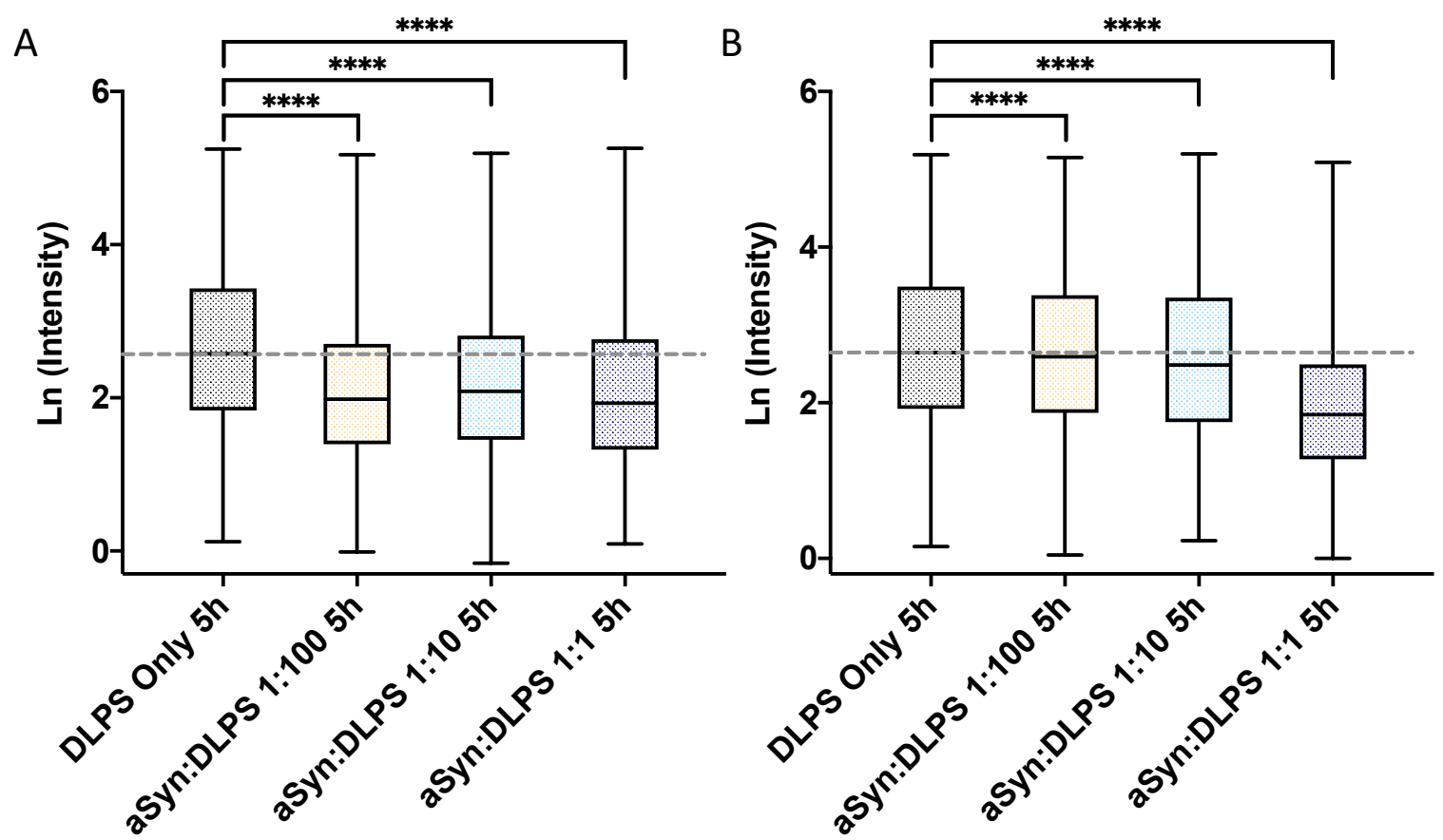

Figure S7: Box and whiskers plot of the scattered intensity distribution following $5 \mathrm{~h}$ of co-incubation with DLPS vesicles for two additional independent replicates. (A) Approximately 16,700 DLPS vesicles in the absence of lipid were tracked and compared with 8,200 (P:L 1:100), 7,800 (P:L 1:10), and $\sim 4,900$ (P:L 1:1) particles. (B) About 15,200 DLPS vesicles in the absence of lipid were tracked and compared with $\sim 16,300$ (P:L 1:100), 10,400 (P:L 1:10), and 4,200 (P:L 1:1) particles. ${ }^{* * * * p}<0.0001$, unpaired two-tailed $t$ test. 




Figure S8: Box and whiskers plots showing scattered intensity distribution per particle for DLPS alone compared with aSyn added at P:L molar ratios of 1:100, 1:10, and 1:1 at $0 \mathrm{~h}, 3 \mathrm{~h}$ and $5 \mathrm{~h} . n s$, not significant, $* * p<0.01, * * * p<0.001$, and $* * * * p<0.0001$ (unpaired two-tailed t test). Dotted line shows the mean scattered intensity per particle for DLPS vesicles in the absence of added protein. All plots shown are representative distributions of a single replicate from three independent replicates. 

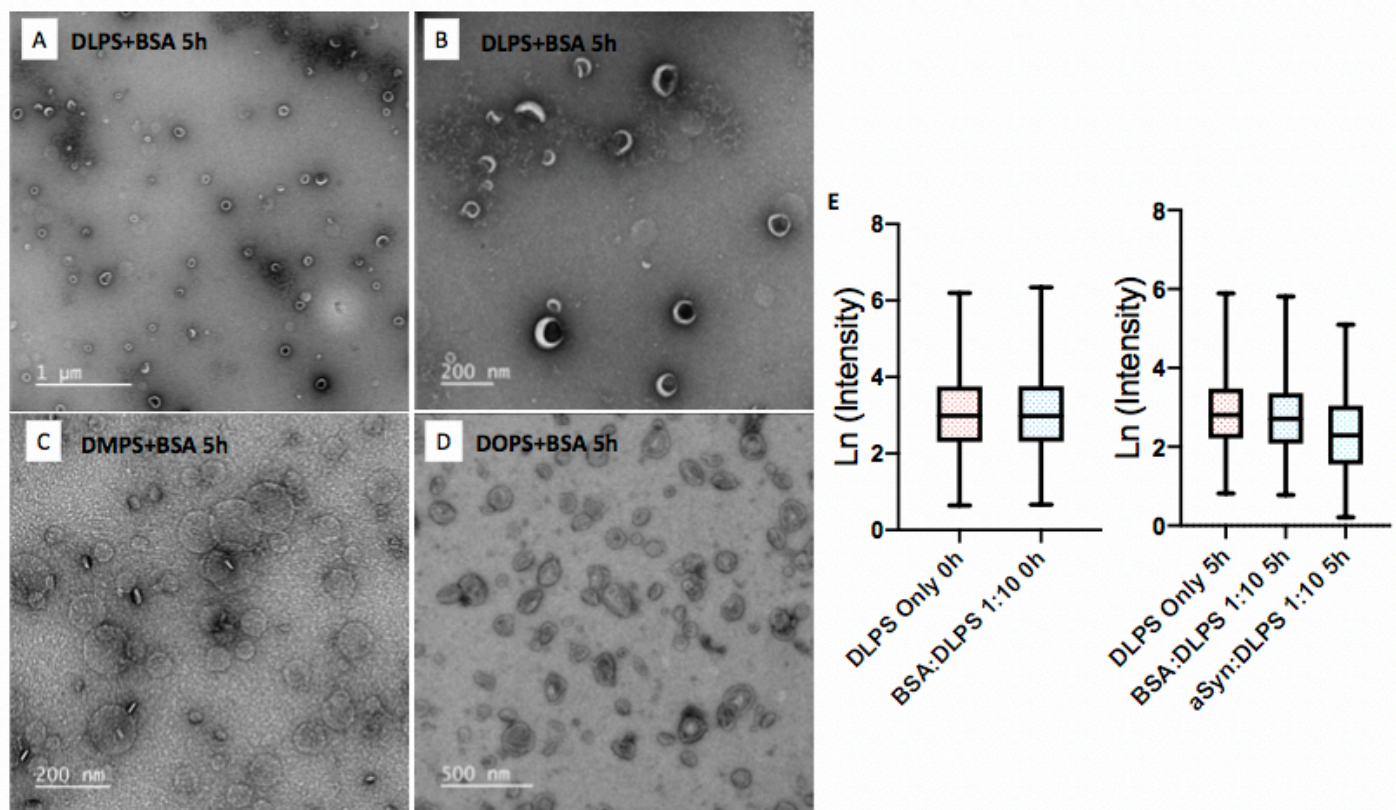

Figure S9: Control experiments with BSA and liposomes. BSA is known to adsorb to PS lipid surfaces and induces dye leakage from vesicles ${ }^{30}$. BSA and DOPS, DMPS or DLPS LUVs were incubated in PBS at 1:10 P:L molar ratio. (A-D) Representative TEM images showing that vesicles largely retained their spherical shape and size following incubation with BSA. No tubules, helical ribbons or coils were observed with DLPS. (E) Scattered intensity per particle with DLPS alone or incubated with BSA or aSyn at 1:10 P:L molar ratio of 1:10. There is no statistically significant change in the mean intensity of the distribution with addition of BSA, in contrast to the change with addition of aSyn. We conclude that the extensive membrane remodeling of DLPS is specific to aSyn. 

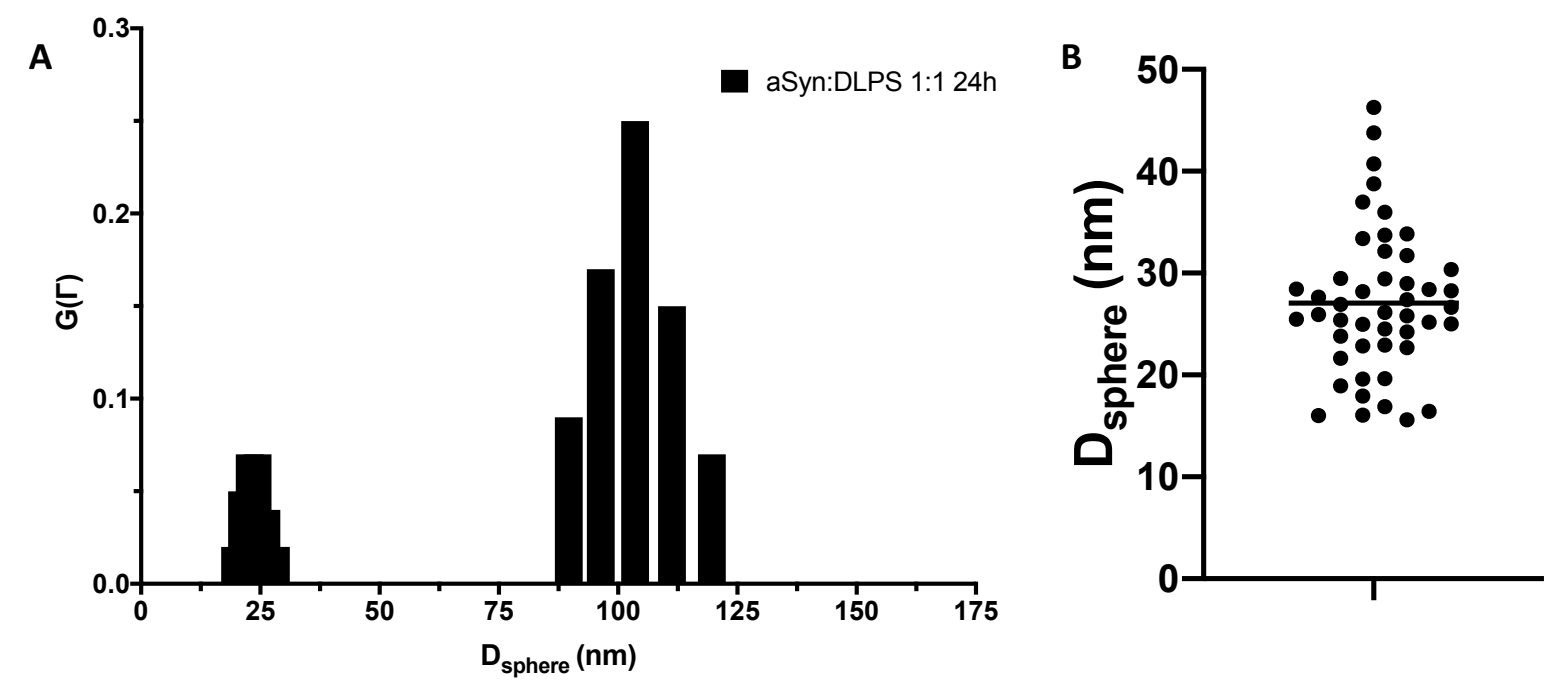

Figure S10: DLS of aSyn incubated with DLPS at a 1:1 P:L molar ratio for $24 \mathrm{~h}$. (A) CONTIN analysis of the scattering size distribution, showing a bimodal distribution centered at $\sim 25 \mathrm{~nm}$ and $\sim 100 \mathrm{~nm}$. (B) Hydrodynamic diameter of discoid particles measured by TEM using equation S7, demonstrating consistency between DLS and TEM estimates of discoid particle size of $\sim 25 \mathrm{~nm}$. The discoids are too small to be detected by NTA. The peak detected by DLS centered at $100 \mathrm{~nm}$ is consistent with NTA particle size as well as estimates of $D_{\text {sphere }}$ for tubules from TEM images. 

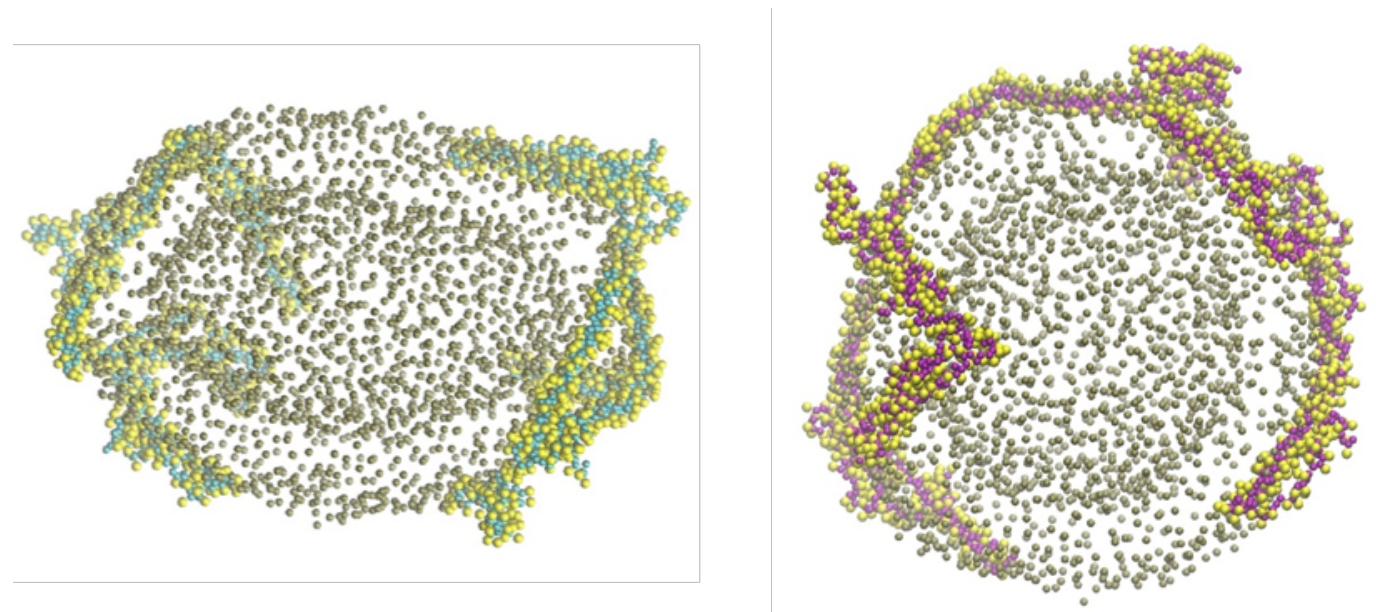

Figure S11. Snapshots from simulations of 10 aSyn monomers after adsorption to DLPS (left) and DOPS (right) vesicles, respectively, at $360 \mathrm{~K}$ using the standard MARTINI force field with antifreeze water beads. The aSyn monomers elongate the DLPS vesicle and accumulate near the poles, whereas the DOPS vesicle remains spherical. These results are consistent with observations from the BMW MARTINI force field. 

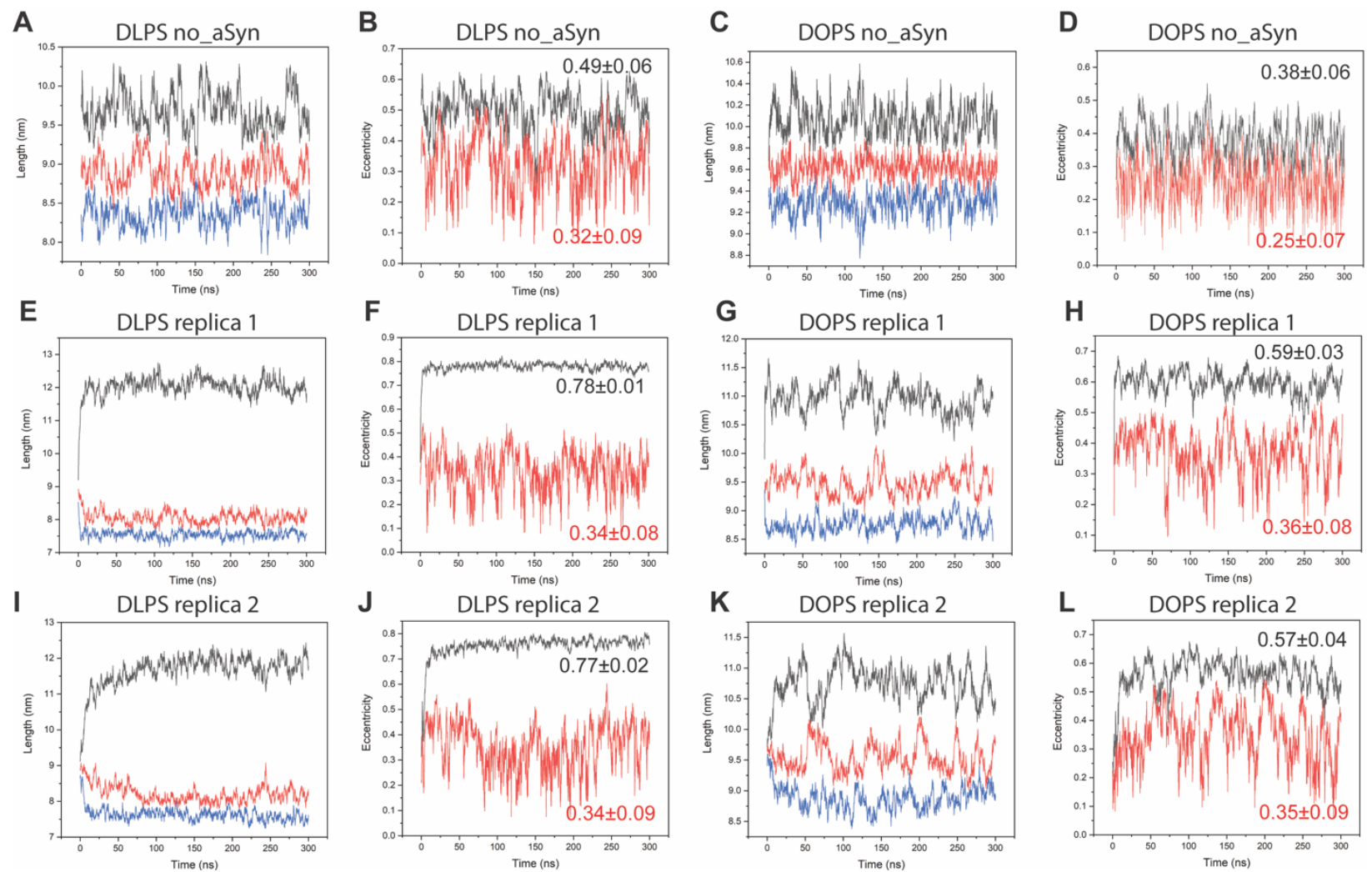

Figure S12: (A) Lengths of the three principle axes of a DLPS vesicle in the absence of aSyn as a function of time in ns. The black, red, and blue curves correspond to the longest, median, and the shortest axes, respectively. (B) Changes of meridional (black curve) and equatorial (red curve) eccentricities of a DLPS vesicle in the absence of aSyn as a function of time in ns. (C), (E), (G), (I), (J) are changes of three principle axes of a DOPS vesicle in the absence of aSyn, a DLPS vesicle in the presence of 10 aSyn, a DOPS vesicle in the presence of 10 aSyn, a second DLPS vesicle in the presence of 10 aSyn, a second DOPS vesicle in the presence of 10 aSyn, respectively. (D), (F), (H), (J), and (L) are changes of meridional and equatorial eccentricities of a DOPS vesicle in the absence of aSyn, a DLPS vesicle in the presence of 10 aSyn, a DOPS vesicle in the presence of $10 \mathrm{aSyn}$, a second DLPS vesicle in the presence of 10 aSyn, a second DOPS vesicle in the presence of 10 aSyn, respectively. 


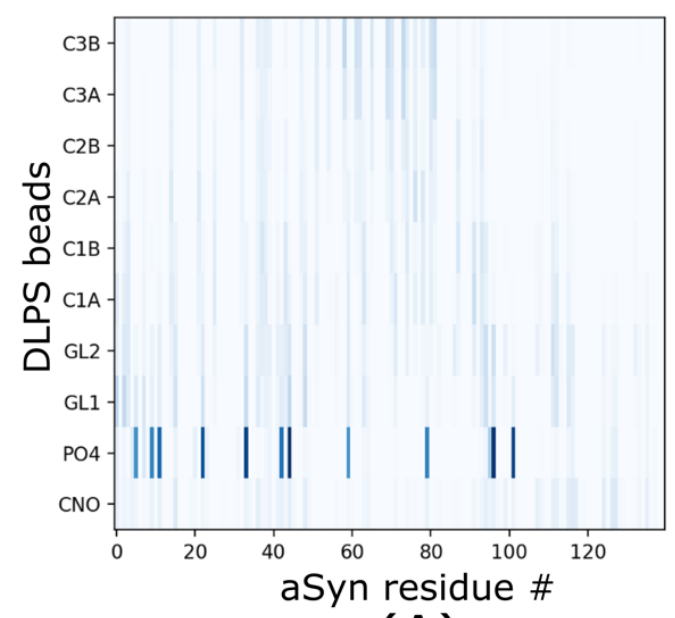

(A)

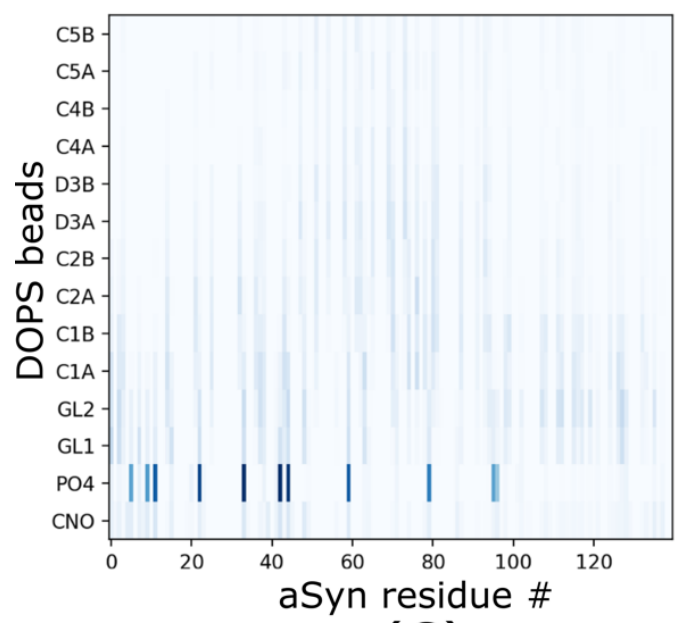

(C)
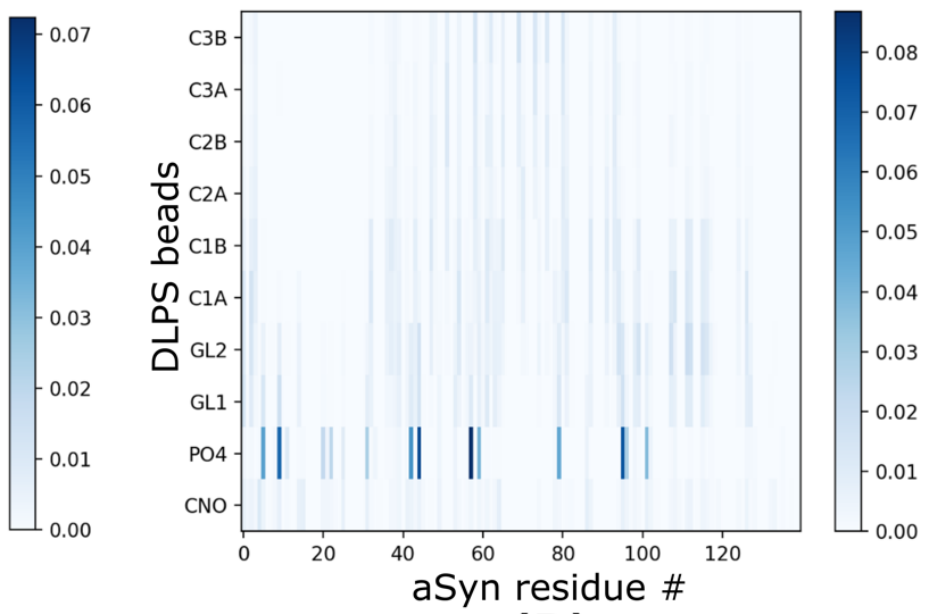

(B)

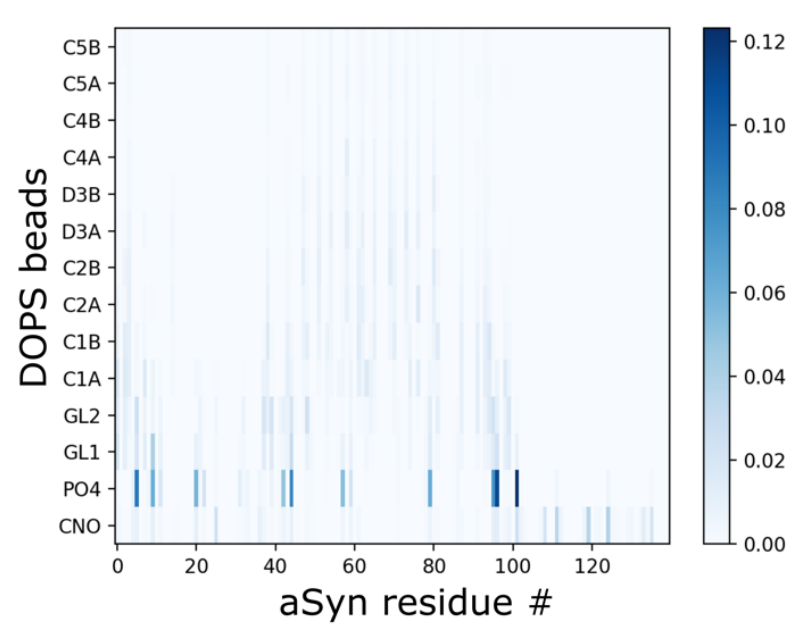

(D)

Figure 13. Contact maps for interactions between aSyn side chain beads and lipid beads. The color bar indicates the time-averaged number of contacts, with larger values indicating a higher likelihood that two beads are in contact. (A) and (B) show contacts with DLPS bilayers from two independent simulations. (C) and (D) show contacts with DOPS bilayers from two independent simulations. In all cases, contacts are primarily between negatively charged PO4 beads in the lipids and positively charged aSyn residues. 

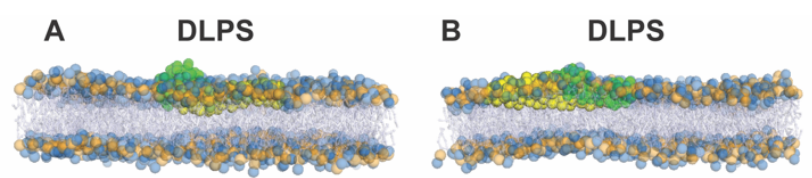

E

$\mathbf{F}$
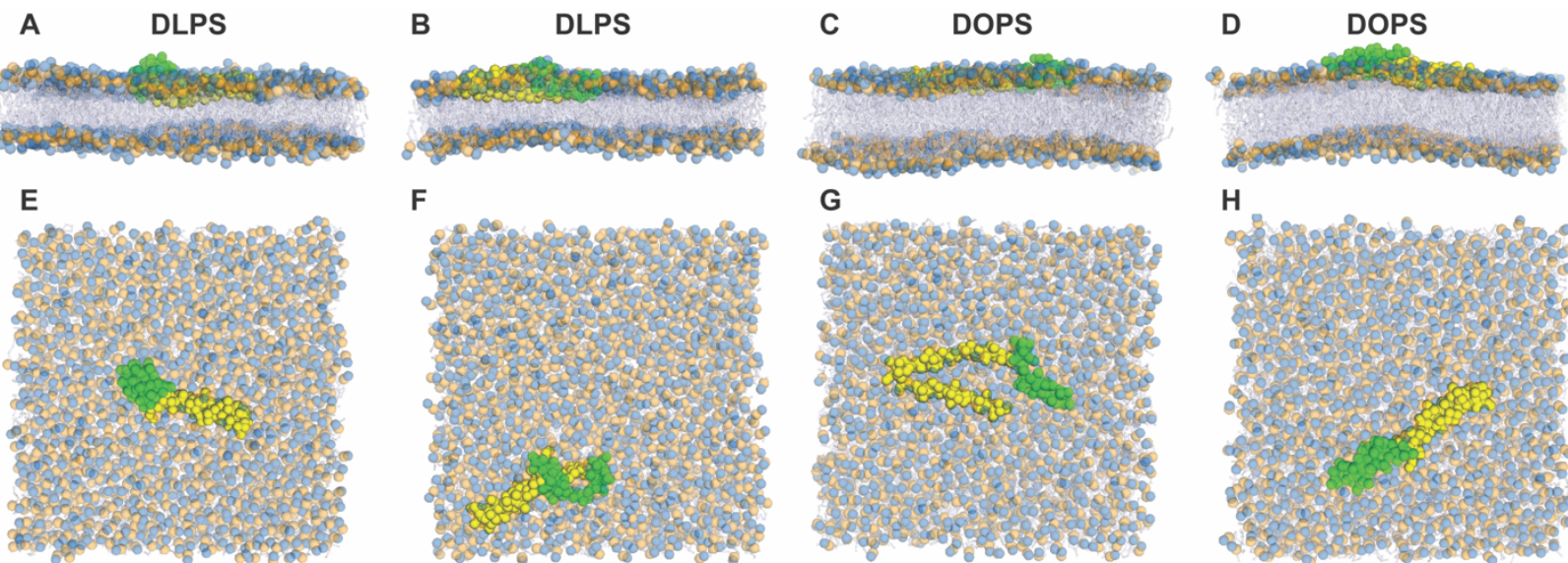

G
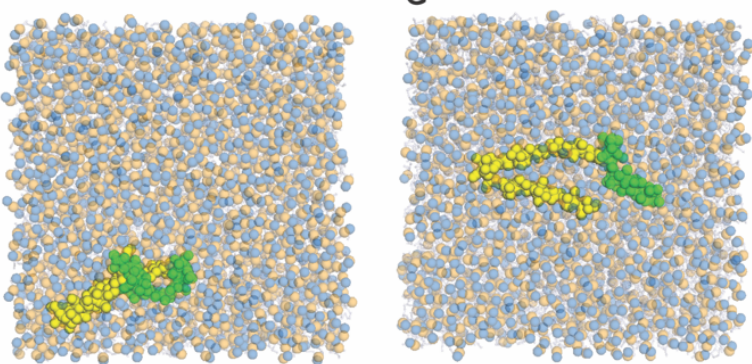

H
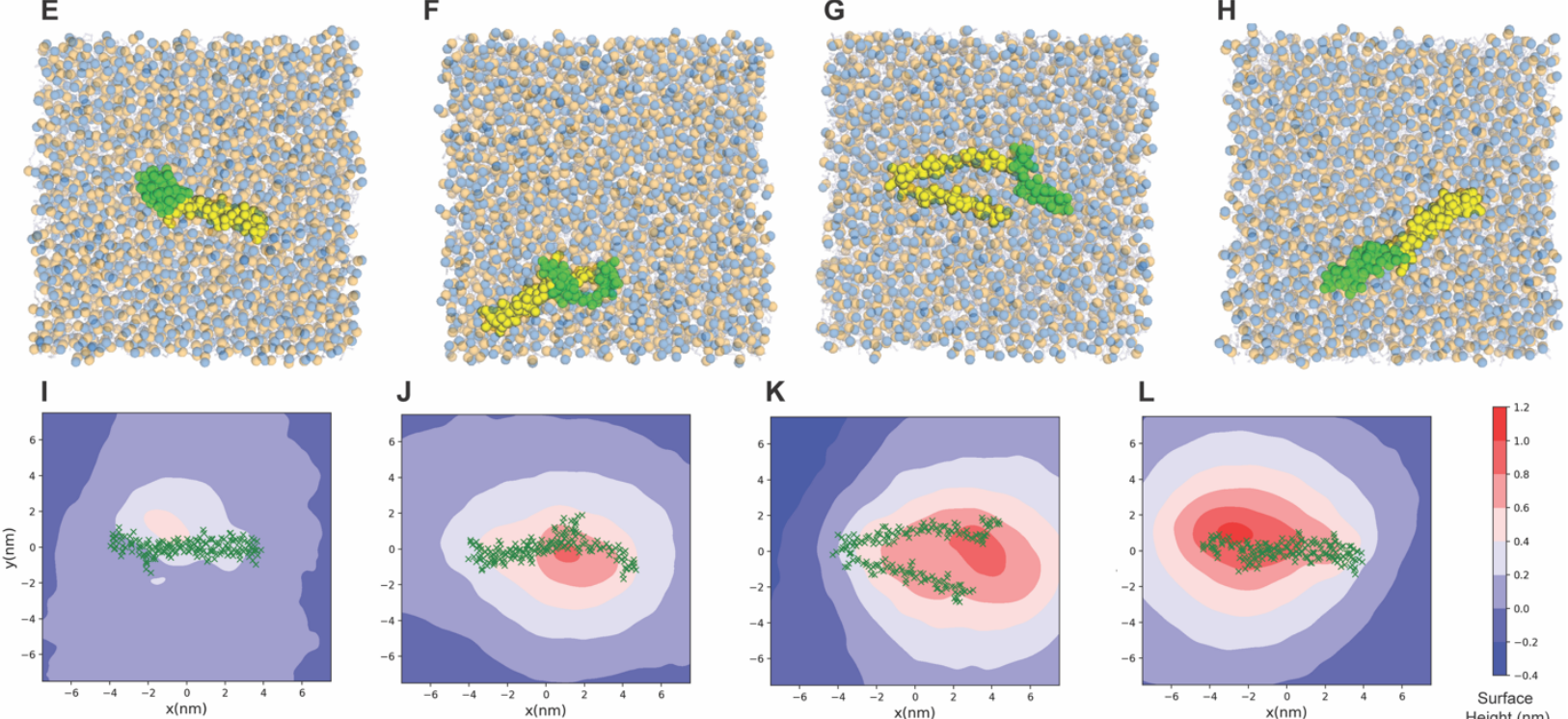

$\mathbf{K}$

\section{L}
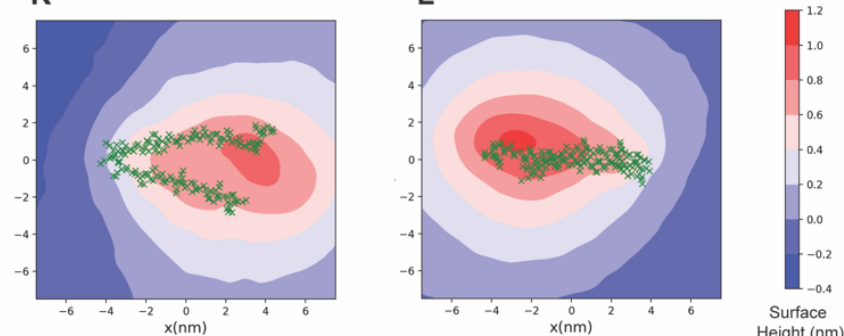

Figure S14: Side view (A-D) and top-down (E-H) view of the two replicas of the DLPS (A, B, E, F) and DOPS (C, D, G, H) bilayer systems. For clarity, only headgroup beads of DOPS or DLPS are shown (phosphate in orange and serine in blue). The first 99 amino acids of aSyn are shown in yellow and the tail beads are in green. (I) and (J) show time-averaged height contours indicating deviations in the height of the bilayer midplane at each $x-y$ position relative to the average height of the bilayer midplane.

Contours are shown for the two DLPS bilayer replicas. The projected positions of the first 99 amino acids (the first 190 beads) of aSyn are displayed as green crosses using the positions from the final configuration of the production simulation. The $x$-axis is aligned with the long axis of the first 99 amino acids. (K) and (L) show averaged height contours indicating deviations in the height of the bilayer midplane for the two DOPS bilayer replicas. The color bar is the same for (I)-(L). 

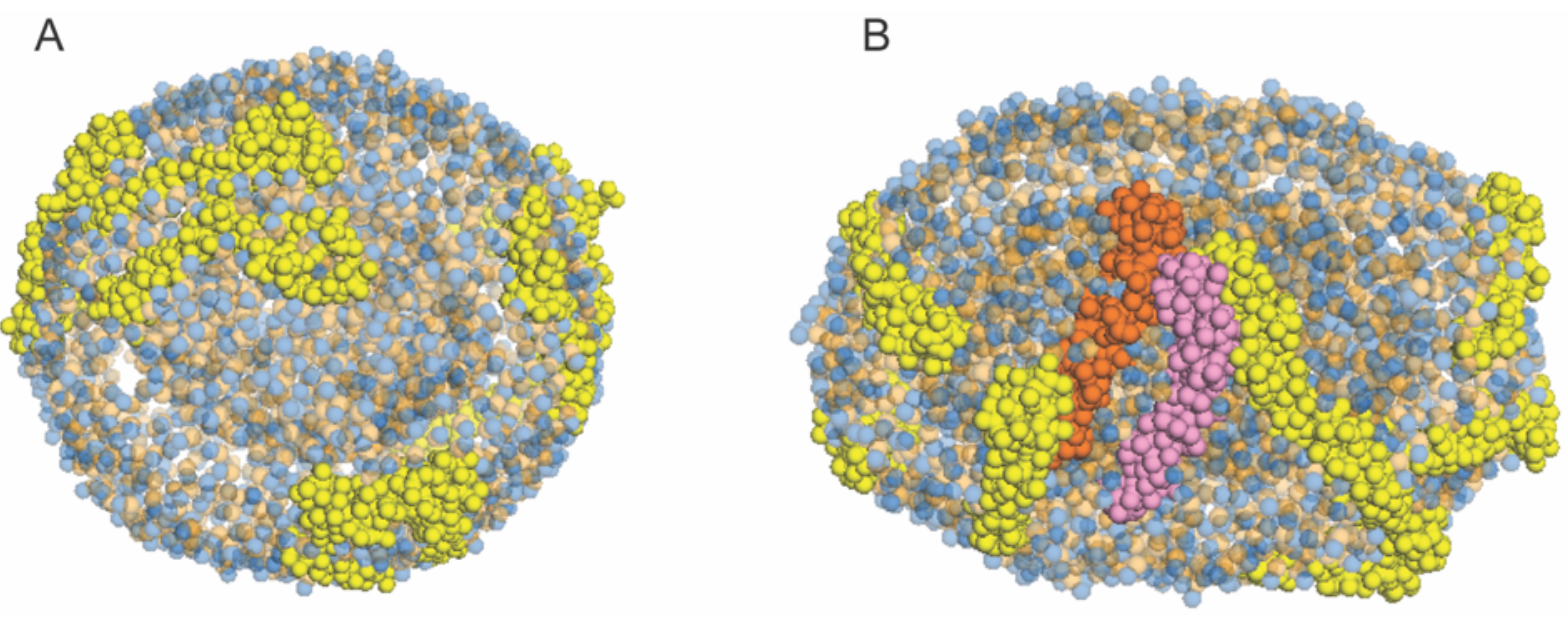

C
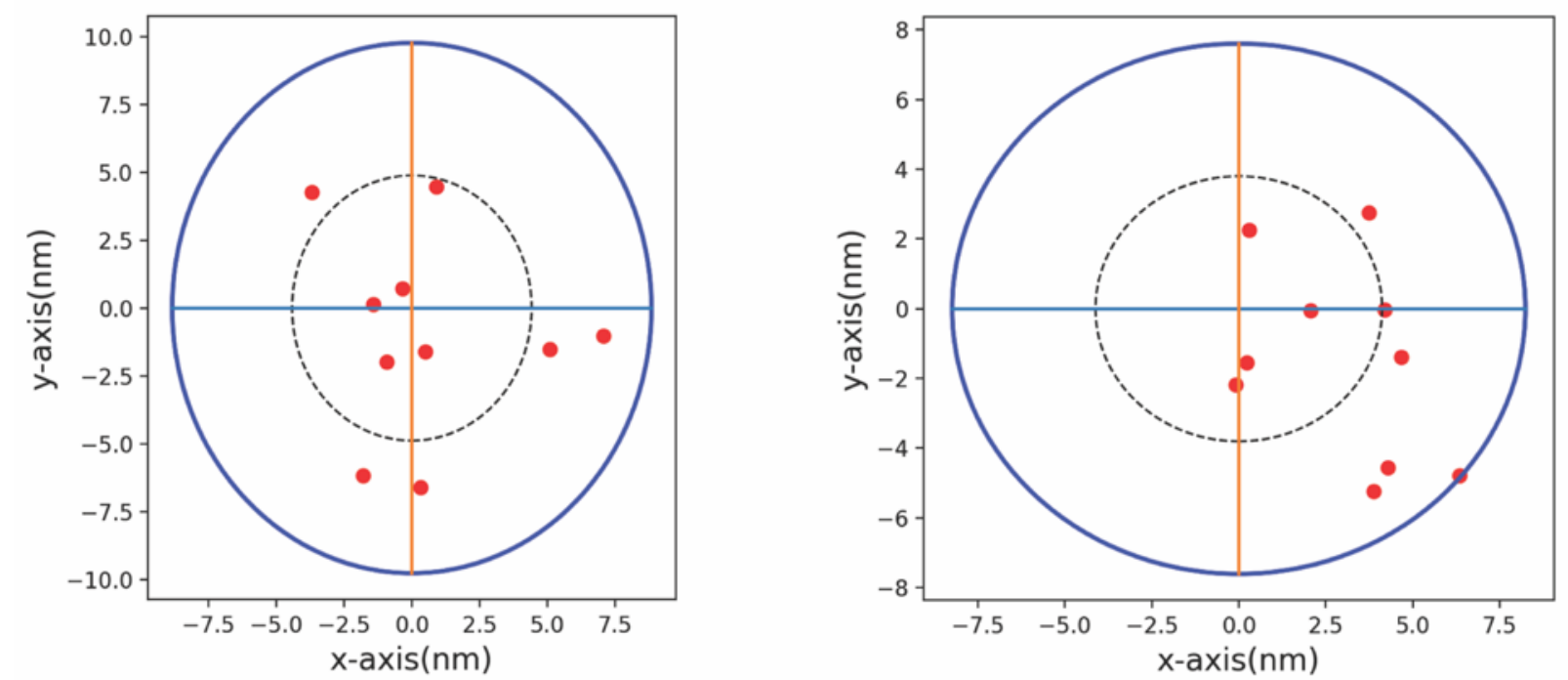

Figure S15: (A) Snapshot of the second replica of a DOPS vesicle with 10 aSyn monomers after the production simulation. (B) Snapshot of the second replica of a DLPS vesicle with 10 aSyn monomers after the production simulation. (C-D) Stereographic projections of the location of the 10 aSyn monomers with respect to the equatorial plane for the simulation snapshots in (A and $\mathrm{B})$. 
A
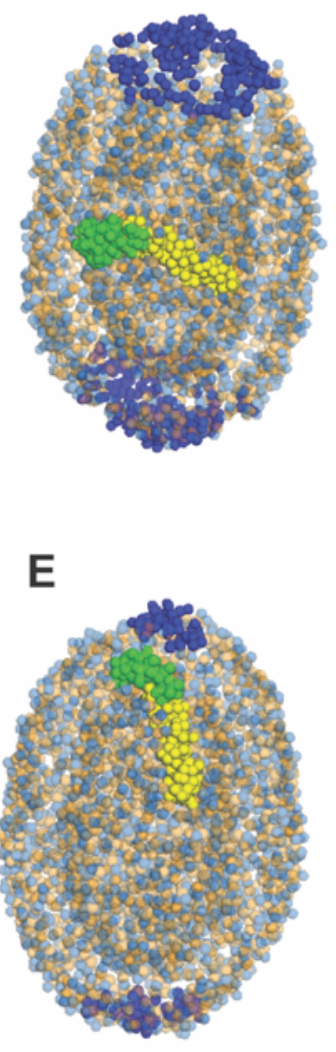

B
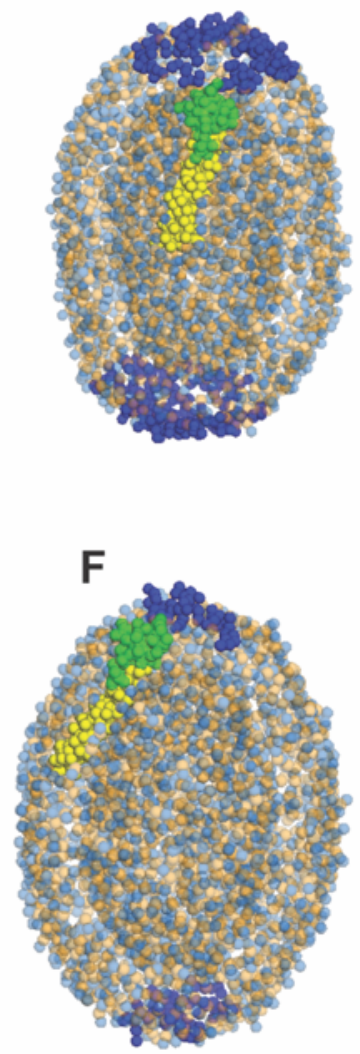
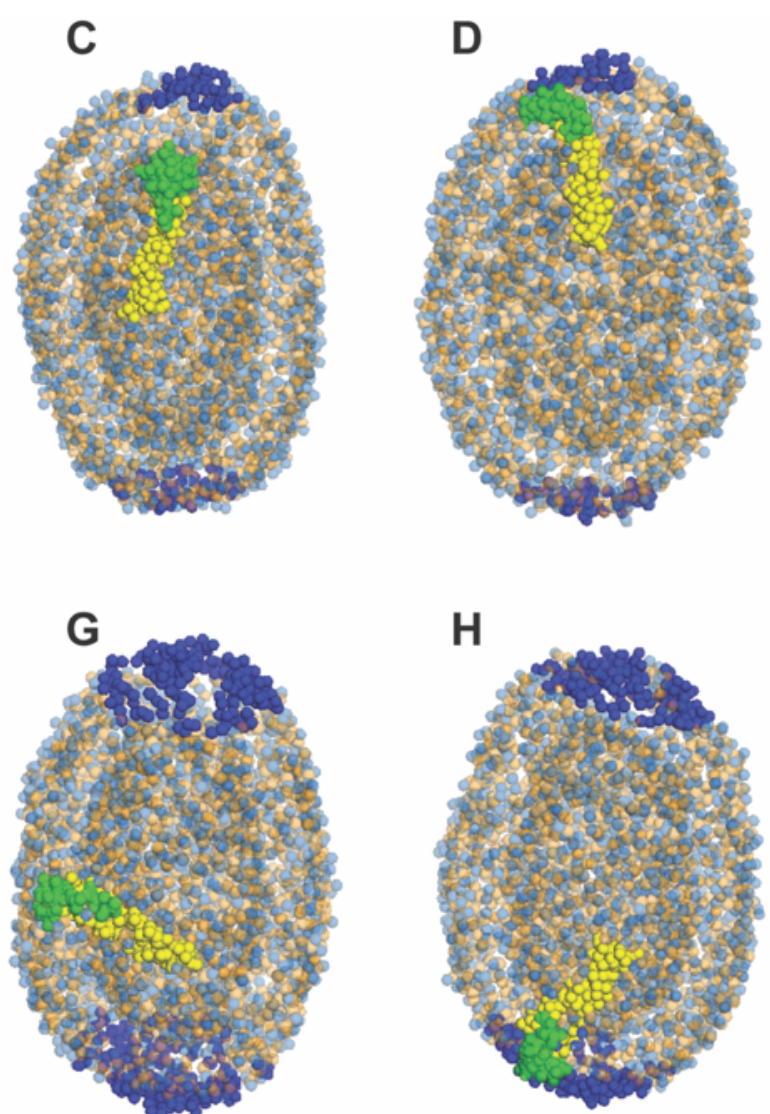

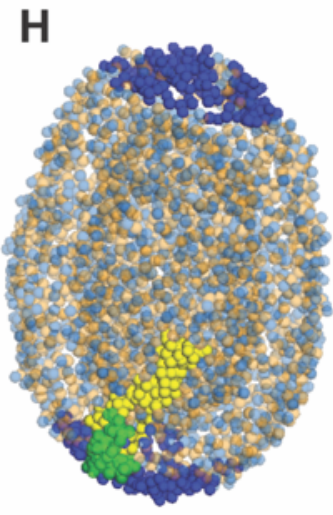

Figure S16: (A) Snapshot of the initial position of an aSyn monomer on an elongated DLPS vesicle (extracted from the second simulation replica of the DLPS vesicle with 10 aSyn monomers and drawn as the pink monomer in Figure S15. 100 phosphate beads are restrained at each pole and indicated in blue. (B) Snapshot of final position of the aSyn monomer on the elongated vesicle. (C) Same configuration as (B) but with only 30 restrained phosphate beads. This configuration was used as the initial position for a second simulation. (D) Snapshot of final position of this aSyn monomer from the second simulation. (E) Same configuration as (D) but with only 24 restrained phosphate beads, which was used as the initial position for a third simulation. (F) Snapshot of the final position of the aSyn monomer from the third simulation. (G) Snapshot of the initial position of an aSyn monomer on an elongated DLPS vesicle (extracted from the second simulation replica of the DLPS vesicle with 10 aSyn monomers and drawn as the orange monomer in Figure S15. 100 phosphate beads are restrained at each pole and indicated in blue. (H) Snapshot of final position of the aSyn monomer from $(\mathrm{G})$ on the elongated vesicle. 

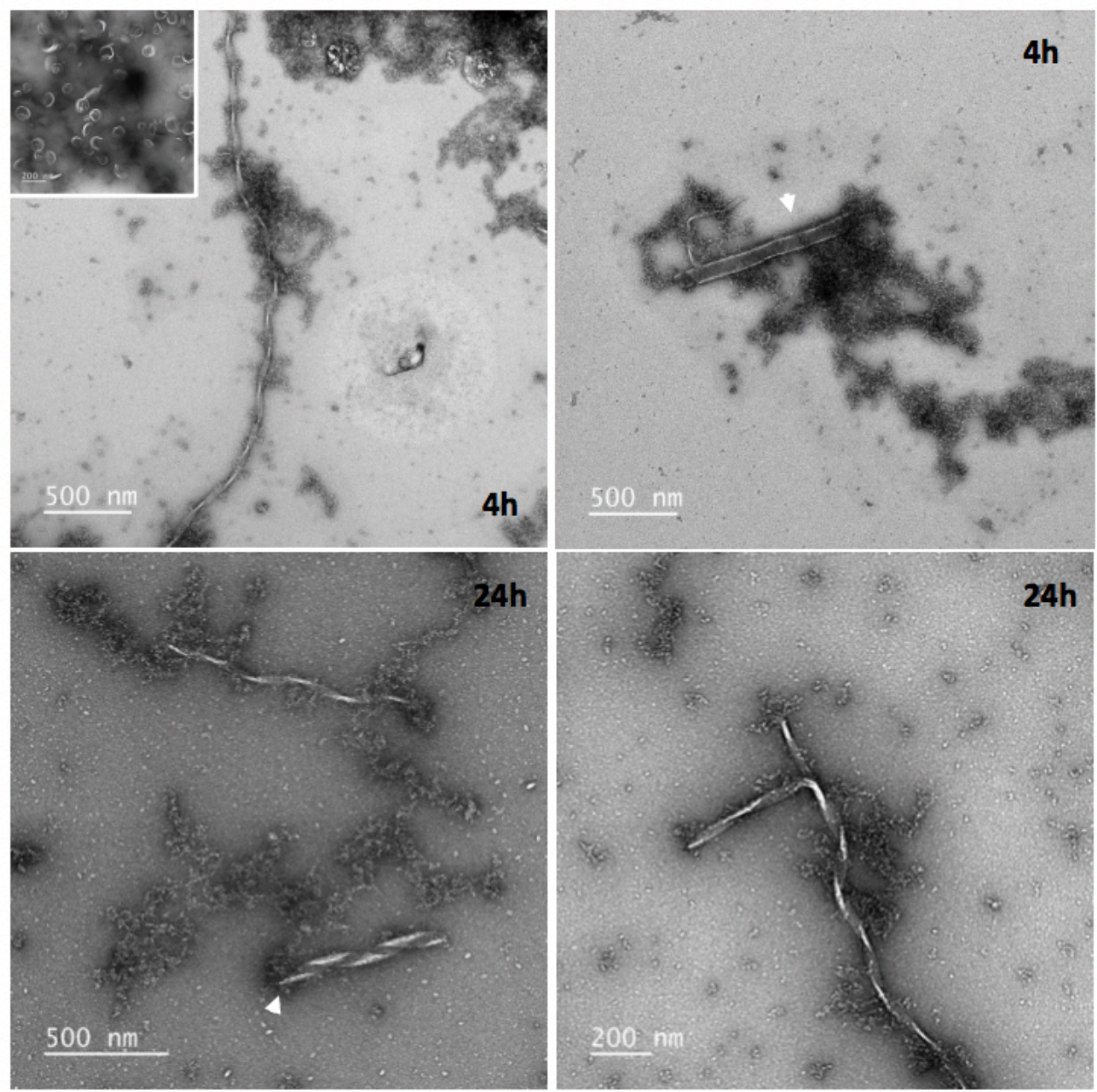

Figure S17: Additional TEM images revealing the presence of twisted and helical ribbons and nanotubes (arrow heads) after incubation of aSyn with DLPS vesicles. Samples were prepared at 1:10 P:L molar ratio in $\mathrm{PB}$ at $30^{\circ} \mathrm{C}$ and imaged after $4 \mathrm{~h}$ (top panel) and $24 \mathrm{~h}$ (bottom panel). Inset shows lipid only control after $4 \mathrm{~h}$ at $30^{\circ} \mathrm{C}$. 

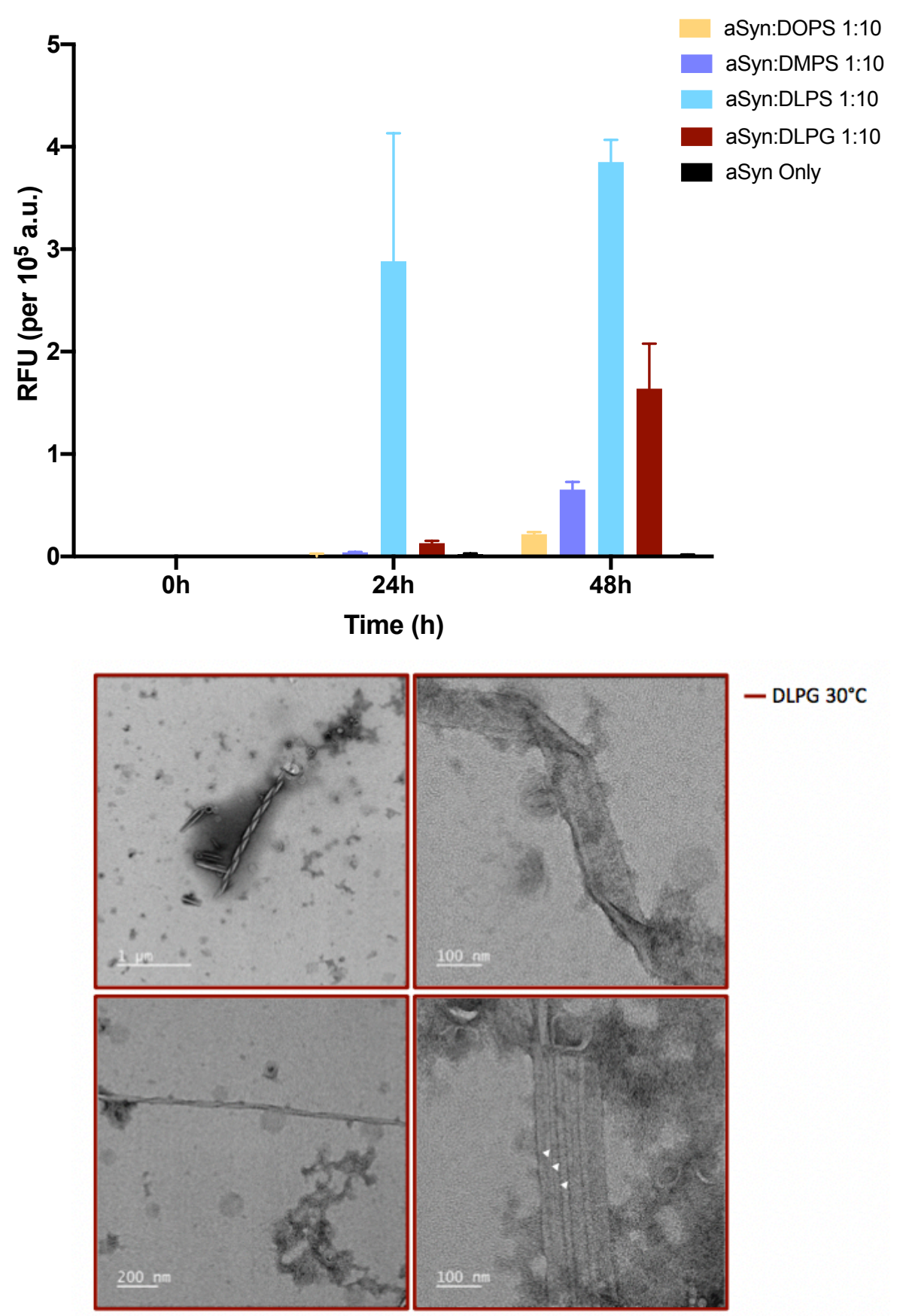

Figure S18: Aggregation of aSyn in the presence of DOPS, DMPS, DLPS, or DLPG vesicles $(80 \mu \mathrm{M}$ aSyn: $800 \mu \mathrm{M}$ lipid), with incubation at $30^{\circ} \mathrm{C}$ in PB. (A) ThT fluorescence measurement of kinetics of amyloid formation. (B) Representative TEM images of aSyn with DLPG vesicles following $72 \mathrm{~h}$ of incubation, revealing coiled ribbons and wide $(\sim 100 \mathrm{~nm})$ sheets. White arrows indicate appearance of a lamellar pattern ${ }^{31}$. 


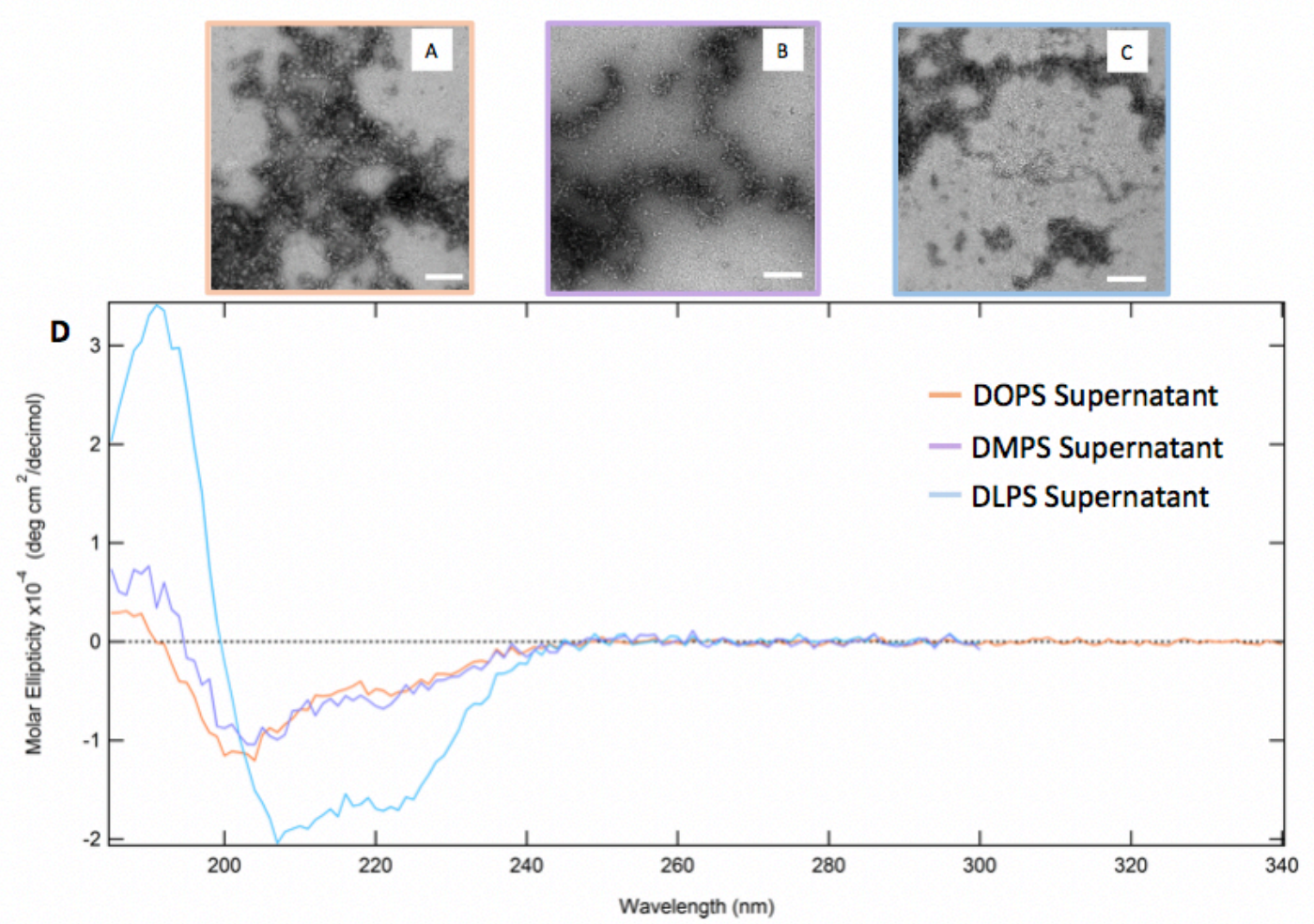

Figure S19: Analysis of supernatant, prepared by incubation at 1:10 P:L molar ratio in $\mathrm{PB}$ at $30^{\circ} \mathrm{C}$ for 48 $\mathrm{h}$ followed by separation by ultracentrifugation. The distribution of protein and lipid in supernatant and pellet is shown in Table S4. (A) Representative TEM image of supernatant of mixtures of aSyn and DOPS, revealing examples of what appears to be lysed membranes. Approximately $50 \%$ of protein and $26 \%$ of lipid was recovered in supernatant. Scale bars are $50 \mathrm{~nm}$. (B) Representative TEM image of supernatant of mixtures of aSyn and DMPS. Approximately $29 \%$ of protein and negligible lipid was recovered in supernatant. (C) Representative TEM image of supernatant of mixtures of aSyn and DLPS, showing an example of tubule formation and lysed membranes. Approximately $90 \%$ of protein and lipid was recovered in supernatant. (D) Circular dichroism spectra of supernatants, indicating disordered conformations for aSyn with DOPS and DMPS and $\alpha$-helical secondary structure of aSyn in the DLPS supernatant. 


\section{Supplemental References}

1. Ferdani, R.; Li, R.; Pajewski, R.; Pajewska, J.; Winter, R. K.; Gokel, G. W. Transport of chloride and carboxyfluorescein through phospholipid vesicle membranes by heptapeptide amphiphiles. Org. Biomolec. Chem. 2007, 5, 2423.

2. Bergstrom, C. L.; Beales, P. A.; Lv, Y.; Vanderlick, T. K.; Groves, J. T. Cytochrome c causes pore formation in cardiolipin-containing membranes. Proc. Natl. Acad. Sci. U.S.A. 2013, 110:62696274.

3. Pecora, R. Dynamic light scattering measurement of nanometer particles in liquids. J. Nanoparticle Res. 2000, 2:123-131.

4. Stewart, J. C. M. Colorimetric determination of phospholipids with ammonium ferrothiocyanate. Anal. Biochem. 1980, 104:10-14.

5. Abraham, M. J.; Murtola, T.; Schulz, R.; Páll, S.; Smith, J. C.; Hess, B.; Lindahl, E. GROMACS: High performance molecular simulations through multi-level parallelism from laptops to supercomputers. SoftwareX. 2015, 1-2:19-25.

6. Ulmer, T. S.; Bax, A.; Cole, N. B.; Nussbaum, R. L. Structure and dynamics of micelle-bound human $\alpha$-synuclein. J. Biol. Chem. 2005, 280:9595-9603.

7. Yesylevskyy, S. O.; Schäfer, L. V.; Sengupta, D.; Marrink, S. J.; Schaefer, L. V.; Sengupta, D.; Marrink, S. J. Polarizable water model for the coarse-grained MARTINI force field. PLoS Comp. Biol., 2010, 6,1-17.

8. Sheavly, J.K.; Pedersen, J.A.; Van Lehn, R.C. Curvature-driven adsorption of cationic nanoparticles to phase boundaries in multicomponent lipid bilayers. Nanoscale 2019, 11, 27672778 .

9. Das, M.; Dahal, U.; Mesele, O.; Liang, D.; Cui, Q. Molecular dynamics simulation of interaction between functionalized nanoparticles with lipid membranes: Analysis of coarse-grained models. $J$. Phys. Chem. B. 2019, 123, 10547-1056

10. Wu, Z.; Cui, Q.; Yethiraj, A. A new coarse-grained force field for membrane-peptide simulations. J. Chem. Theory Comput. 2011, 7, 3793-3802.

11. Rawickz, W.; Olbrich, K. C.; McIntosh, T.; Needham, D.; Evans, E. Effect of chain length and unsaturation on elasticity of lipid bilayers. Biophys. J. 2000, 79:328-339.

12. Berendsen, H. J. C.; Postma, J. P. M.; van Gunsteren, W. F.; DiNola, A.; Haak, J. R. Molecular dynamics with coupling to an external bath. J. Chem. Phys. 1984, 81:3684-3690.

13. Nosé, S. A molecular dynamics method for simulations in the canonical ensemble. Molec. Physics. 1984, 52:255-268.

14. Hoover, W. G. Canonical dynamics: Equilibrium phase-space distributions. Physical Review A. 1985, 31:1695-1697

15. Parrinello, M.; Rahman, A. Polymorphic transitions in single crystals: A new molecular dynamics method. J. Appl. Phys. 1981, 52:7182-7190.

16. Nosé, S.; Klein, M. L. Constant pressure molecular dynamics for molecular systems. Molecular Physics. 1983, 50:1055-1076.

17. Watson, M. C.; Brandt, E. G.; Welch, P. M.; Brown, F. L. H. Determining biomembrane bending rigidities from simulations of modest size. Phys. Rev. Lett. 2012, 10.1103/PhysRevLett. 109.028102

18. Matsuzaki, K.; Murase, O.; Sugishita, K.; Yoneyama, S.; Akada, K.; Ueha, M.; Nakamura, A.; Kobayashi, S. Optical characterization of liposomes by right angle light scattering and turbidity measurement. Biochim. Biophys. Acta (BBA) - Biomem. 2000, 1467:219-226.

19. Gallego-Urrea, J. A.; Tuoriniemi, J.; Hassellöv, M. Applications of particle-tracking analysis to the determination of size distributions and concentrations of nanoparticles in environmental, biological and food samples. TrAC Trends Anal. Chem. 2011, 30:473-483.

20. Jennings, B. R.; Parslow, K. Particle size measurement: The equivalent spherical diameter. Proc. Royal Soc. A: Math. Phys. Eng. Sci. 1988, 419:137-149. 
21. Pedersen, J. S. Analysis of small-angle scattering data from colloids and polymer solutions: modeling and least-squares fitting. Adv. Coll. Interface Sci. 1997, 70:171-210.

22. Johnsson, M.; Edwards, K. Liposomes, disks, and spherical micelles: Aggregate atructure in mixtures of gel phase phosphatidylcholines and poly(ethylene glycol)-phospholipids. Biophys. J. 2003, 85:3839-3847.

23. Mazer, N. A.; Benedek, G. B.; Carey, M. C. Quasielastic light-scattering studies of aqueous biliary lipid systems. Mixed micelle formation in bile salt-lecithin solutions. Biochem. 1980, 19:601-615.

24. Amorós, D.; Ortega, A.; García de la Torre, J. Hydrodynamic properties of wormlike macromolecules: Monte Carlo simulation and global analysis of experimental data. Macromol. 2011, 44:5788-5797.

25. Yamakawa, H.: Fujii, M. Translational friction coefficient of wormlike chains. Macromol. 1973, 6:407-415.

26. Koyama, R. Light scattering of stiff chain polymers. J. Phys. Soc. Japan. 1973, 34:1029-1038.

27. Shen, C. L.; Fitzgerald, M. C.; Murphy, R. M. Effect of acid predissolution on fibril size and fibril flexibility of synthetic beta-amyloid peptide. Biophys. J. 1994, 67:1238-1246.

28. Tsay, J. M.; Doose, S.; Weiss, S. Rotational and translational diffusion of peptide-coated $\mathrm{CdSe} / \mathrm{CdS} / \mathrm{ZnS}$ nanorods studied by fluorescence correlation spectroscopy. J. Am. Chem. Soc. 2006, 128:1639-1647.

29. Lu, K.; Jacob, J.; Thiyagarajan, P.; Conticello, V. P.; Lynn, D. G. Exploiting amyloid fibril lamination for nanotube self-assembly. J. Am. Chem. Soc. 2003, 125:6391-6393.

30. Schroit, A. J.; Fidler, I. J. Effects of liposome structure and lipid composition on the activation of the tumoricidal properties of macrophages by liposomes containing muramyl dipeptide. Cancer Res. 1982, 42:161-167.

31. Pallbo, J.; Sparr, E.; Olsson, U. Aggregation behavior of the amyloid model peptide NACore. Quart. Rev. Biophys. 2019, 10.1017/S0033583519000039. 